\title{
REGULAR SEMIGROUPS, FUNDAMENTAL SEMIGROUPS AND GROUPS
}

\author{
D. B. MCALISTER
}

(Received 22 October 1979)

Communicated by T. E. Hall

\begin{abstract}
In this paper we obtain necessary and sufficient conditions on a regular semigroup in order that it should be an idempotent separating homomorphic image of a full subsemigroup of the direct product of a group and a fundamental or combinatorial regular semigroup. The main tool used is the concept of a prehomomorphism $\theta: S \rightarrow T$ between regular semigroups. This is a mapping such that $(a b) \theta \leqslant a \theta b \theta$ in the natural partial order on $T$.
\end{abstract}

1980 Mathematics subject classification (Amer. Math. Soc.) : $20 \mathrm{M} 10$.

Much of the structure theory of regular semigroups has been concerned with the problem of determining the extent to which a regular semigroup $S$ can be described in terms of groups and semigroups determined by the idempotents of $S$. If $\rho$ is any congruence on $S$ which is contained in Green's relation $\mathscr{H}$, the $\rho$-classes which contain idempotents are subgroups of $S$ so that $S$ is a coextension of $S / \rho$ by a family of groups. In particular $S$ is a coextension of $F=S / \mu$, where $\mu$ is the maximum congruence on $S$ contained in $\mathscr{H}$, by a family of groups. The semigroup $F$ is fundamental in the sense that no nontrivial congruence on $F$ is contained in $\mathscr{H}$. For this reason fundamental regular semigroups have played an important role in the structure theory of regular semigroups.

Munn(1970) showed that any fundamental inverse semigroup can be realized as a semigroup of isomorphisms between the principal ideals of its semilattice of idempotents. Nambooripad (1979), following Hall (1973), has extended Munn's result to regular semigroups by showing that each fundamental regular semigroup $F$

This research was partially supported by NSF Grant MCS 7607212A01. 
can be realized as a semigroup constructed from partial isomorphisms of the biordered set of idempotents of $F$.

On the other hand, McAlister and Reilly (1977) have shown that any inverse semigroup $S$ is an idempotent separating homomorphic image of a subdirect product of a fundamental inverse semigroup $F$ and a group $G$. The kernel of this homomorphism is a semilattice of groups so that $S$ is obtained from a fundamental inverse semigroup and a group (thus from its semilattice and a group) by factoring out subgroups.

The main purpose of this paper is to obtain necessary and sufficient conditions under which an analogous result holds for regular semigroups. The main tool used to obtain these conditions is the notion of a prehomomorphism which was used in McAlister and Reilly (1977) to characterize $E$-unitary covers, for inverse semigroups. Prehomomorphisms on regular semigroups are introduced in Section 1 and a method for constructing them is developed which is used in Section 2 to obtain necessary and sufficient conditions for a regular semigroup to divide the direct product of a fundamental regular semigroup and a group. These conditions depend on the properties of an equivalence relation $\pi$. A construction for $\pi$ is obtained in Section 3 which is used in Section 4 to show that a regular semigroup $S$ divides the direct product of a fundamental regular semigroup and a group if and only if the smallest full self conjugate subsemigroup $C I G(S)$ is fundamental. The methods used in Sections 2 and 4 can also be applied to determine when a regular semigroup divides the direct product of a combinatorial regular semigroup and a group. This permits us to answer, for regular semigroups, the question of Schützenberger which was considered in McAlister (1980).

\section{Preliminaries}

We shall assume familiarity with the basic results and terminology of semigroup theory which will be found, for example, in Clifford and Preston $(1961,1967)$ or Howie (1976).

A semigroup $S$ is called regular if for each element $a \in S$ there exists $x \in S$ such that $a=a x a$. In this case, there exists $a^{\prime} \in S$ such that both $a=a a^{\prime} a$ and $a^{\prime}=a^{\prime} a a^{\prime}$ are satisfied. Such an element $a^{\prime}$ is called an inverse for $a$; the set of inverses of $a$ is denoted by $V(a)$. Thus $V(a)=\{x \in S: a=a x a, x=x a x\}$.

A regular semigroup is called inverse if each element has a unique inverse. In this case, the inverse of a product $a b$ is the product $b^{\prime} a^{\prime}$ of the inverses $b^{\prime}$ of $b$ and $a^{\prime}$ of $a$ respectively. This fortunate circumstance does not hold for arbitrary regular semigroups. Nambooripad (1980) has introduced the concept of the sandwich set of a pair of idempotents $e, f$ in a regular semigroup $S$. This concept permits one to locate inverses of products in regular semigroups. 
DEFINITION 0.1. Let $S$ be a regular semigroup and let $e, f$ be idempotents of $S$. The sandwich set $S(e, f)$ of $S$ is the set

$$
S(e, f)=\left\{g^{2}=g \in S ; e g f=e f, g e=g=f g\right\} .
$$

Lemma 0.2. (Nambooripad, 1980) Let $S$ be a regular semigroup and let $a, b \in S$. Suppose that $a^{\prime} \in V(a), b^{\prime} \in V(b)$ and let $g \in S\left(a^{\prime} a, b b^{\prime}\right)$. Then $b^{\prime} g a^{\prime} \in V(a b)$.

Furthermore, $a b=a_{1} b_{1}$ where $a_{1}=a g, b_{1}=g b$ and

$$
a b \mathscr{R} a_{1} \mathscr{L} g \mathscr{R} b_{1} \mathscr{L} a b
$$

FitzGerald (1972) has shown that the subsemigroup $I G(S)$, generated by the idempotents of a regular semigroup $S$, is itself regular. Hall (1973) showed that each element of $I G(S)$ can be written as a product of $\mathscr{D}$-equivalent idempotents. This result has been refined by Nambooripad (1979).

If $S$ is a regular semigroup then we shall denote by $\mu$ the maximum congruence on $S$ which is contained in Green's relation $\mathscr{H} . S$ is called fundamental if $\mu$ is the equality relation $\Delta$. Grillet (1974), Hall (1973) and Nambooripad (1979) have given representation theorems for fundamental regular semigroups $S$ as semigroups defined solely in terms of the idempotents of $S$.

There is a minimum group congruence on every regular semigroup. We shall denote this relation by $\sigma$.

LEMMA 0.3 (Nambooripad, 1979). Let $S$ be a regular semigroup. Then each element of $I G(S)$ can be written as a product $e_{1} e_{2} \ldots e_{n}$ where

$$
e_{1} \mathscr{L} e_{2} \mathscr{R} e_{3} \ldots
$$

Inverse semigroups have been extensively studied in recent years and, in this paper, we shall obtain results about regular semigroups by using properties of inverse semigroups. In particular we shall use the fact (Schein (1966)) [see McAlister (1976)] that the set $\mathscr{K}(G)$ of cosets of a group $G$, modulo subgroups of $G$, forms an inverse semigroup under the product

$$
X * Y=\text { smallest coset containing } X Y \text {. }
$$

The natural partial order on $\mathscr{X}(G)$ is the inverse of inclusion.

An inverse semigroup $S$ is called $E$-unitary if the equations $e a=e=e^{2}$ in $S$ together imply that $a^{2}=e$. It is shown in McAlister (1974), that every inverse semigroup $S$ has an $E$-unitary cover $P$ in the sense of the following lemma which summarizes the results on $E$-unitary inverse semigroups which we shall need in this paper. 
LEMMA 0.4 (McAlister (1974) and McAlister and Reilly (1977)). Let $S$ be an inverse semigroup. Then there is an E-unitary inverse semigroup $P$ and an idempotent separating homomorphism of $P$ onto $S ; P$ is called an $E$-unitary cover for $S$.

If $P$ is an E-unitary cover for $S$ then $P$ is a subdirect product of a fundamental inverse semigroup $(S / \mu)$ and a group $G$. Further $G$ is the maximum group homormorphic image of $P$ and, for $(a, g),(b, h) \in P$

$$
(a, g) \sigma(b, h) \text { if and only if } g=h .
$$

\section{Prehomomorphisms}

In a recent paper (1980), K. S. S. Nambooripad has introduced a partial order on an arbitrary regular semigroup which generalizes the natural partial order on an inverse semigroup.

DEFINITION 1.1. Let $S$ be a regular semigroup. Then the relation $\leqslant$ on $S$ defined by $a \leqslant b$ if and only if $a \in b S$ and $a=e b$ for some $e^{2}=e \Re a$

is a partial order on $S$. It is called the natural partial order on $S$.

Although it is not evident from the definition, the definition of the natural partial order is self dual. Further, for $a, b \in S$

$$
a \leqslant a b \quad \text { implies } a=a b
$$

for $a \leqslant a b$ implies $a=e a b$ for some idempotent $e$. Thus $e a=e^{2} a b=e a b=a$ so that $a=e a b=a b$. Dually $a \leqslant b a$ implies $a=b a$.

McAlister (1976) introduced the concept of a prehomomorphism on an inverse semigroup. This notion too can be extended to regular semigroups by means of the natural partial order.

DEFINITION 1.2. Let $S$ and $T$ be regular semigroups. Then a mapping $\varphi: S \rightarrow T$ is $a$ prehomomorphism if $(a b) \varphi \leqslant a \varphi b \varphi$ for all $a, b \in S$.

In McAlister (1976) the definition of a prehomomorphism between inverse semigroups also required that $a \varphi^{-1}=a^{-1} \varphi$ for each $a \in S$. This is in fact a consequence of Definition 1.2. In addition, many of the properties of prehomomorphisms which were obtained for inverse semigroups also hold for regular semigroups. 
LEMMA 1.3. Let $\varphi$ be a prehomomorphism of a regular semigroup $S$ into a regular semigroup $T$ and let $a, b \in S$. Then

(i) $a^{\prime} \varphi \in V(a \varphi)$ for each $a^{\prime} \in V(a)$;

(ii) $\varphi$ maps idempotents of $S$ to idempotents of $T$;

(iii) (ab) $\varphi=a \varphi b \varphi$ if there exist $a^{\prime} \in V(a), b^{\prime} \in V(b)$ such that $a^{\prime} a \omega^{l} b b^{\prime}$ or $b b^{\prime} \omega^{r} a^{\prime} a$;

(iv) $\varphi$ is isotone.

Proof. (i) Let $a^{\prime} \in V(a)$. Then $a \varphi=\left(a a^{\prime} a\right) \varphi \leqslant a \varphi\left(a^{\prime} a\right) \varphi$ and hence $a \varphi=a \varphi\left(a^{\prime} a\right) \varphi$. Further $\left(a^{\prime} a\right) \varphi \leqslant a^{\prime} \varphi a \varphi$ implies $\left(a^{\prime} a\right) \varphi=a^{\prime} \varphi a \varphi e$ for some $e^{2}=e \in T$ so that $a \varphi=a \varphi a^{\prime} \varphi a \varphi e$. This then implies $a \varphi=a \varphi e$ so that $a \varphi=a \varphi a^{\prime} \varphi a \varphi$. Dually

$$
a^{\prime} \varphi=a^{\prime} \varphi a \varphi a^{\prime} \varphi
$$

(ii) Let $e^{2}=e \in S$ and set $a=e \varphi \in T$. Then $a=e \varphi=e^{2} \varphi \leqslant e \varphi e \varphi=a^{2}$, so that $a=a^{2}$.

(iii) Suppose that $a^{\prime} a w^{l} b b^{\prime}$. Then

$$
(a b) \varphi \leqslant a \varphi b \varphi=\left(a b b^{\prime}\right) \varphi b \varphi \text {. }
$$

But $\left(a b b^{\prime}\right) \varphi \leqslant(a b) \varphi b^{\prime} \varphi$ so that $\left(a b b^{\prime}\right) \varphi=(a b) \varphi b^{\prime} \varphi . e$ for some $e^{2}=e \mathscr{L}\left(a b b^{\prime}\right) \varphi$. Thus

$$
(a b) \varphi \leqslant a \varphi b \varphi=\left(a b b^{\prime}\right) \varphi b \varphi=(a b) \varphi b^{\prime} \varphi \cdot e(b \varphi)
$$

so that $(a b) \varphi=(a b) \varphi b^{\prime} \varphi . e(b \varphi)$ and therefore $(a b) \varphi=a \varphi b \varphi$.

A similar argument shows the truth of (iii) if $b b^{\prime} \omega^{r} a^{\prime} a$.

(iv) Let $a, b \in S$ and suppose $a \leqslant b$. Then $a \in b S, a=e b$ for some $e^{2}=e \mathscr{R} a$. Hence, if $a=b x$, we have, firstly,

$$
a \varphi=(b x) \varphi \leqslant b \varphi x \varphi \quad \text { which implies } a \varphi \in b \varphi x \varphi T \subseteq b \varphi T .
$$

Next $a=e b$ with $e \Re d a$ implies $a \varphi=(e b) \varphi \leqslant e \varphi b \varphi$ so that $a \varphi=f(e \varphi b \varphi)$ for some $f^{2}=f \in T$ with $f \mathscr{R} a \varphi$. But aRe implies $e=a a^{\prime}$ with $a^{\prime} \in V(a)$ and, by (iii), since $a^{\prime} a=b b^{\prime}$ where $b=a^{\prime}, b^{\prime}=a, e \varphi=\left(a a^{\prime}\right) \varphi=a \varphi a^{\prime} \varphi$ with $a^{\prime} \varphi \in V(a \varphi)$. Thus $a \varphi \mathfrak{R e} \varphi \mathscr{R} f$ so that $f(e \varphi)=e \varphi$ and $a \varphi=e \varphi b \varphi$ with $e \varphi \mathscr{R} a \varphi$. Hence $a \varphi \leqslant b \varphi$.

COROLlaRY 1.4. Let $\varphi$ be a prehomomorphism of a regular semigroup $S$ into a regular semigroup $T$. Then

(i) $a \in b S$ implies $a \varphi \in b \varphi T$; $a \in S b$ implies $a \varphi \in T(b \varphi)$;

(ii) $\varphi$ preserves Green's relations; if $\mathscr{X}$ is any one of Green's relations the a $\mathscr{K b}$ implies a $\boldsymbol{x} \boldsymbol{X} b \varphi$

(iii) $\varphi$ preserves the quasiorders $\omega^{l}$ and $\omega^{r}$ on the idempotents of $S$.

COROLlaRy 1.5. The composition of prehomomorphisms is a prehomomorphism. Thus regular semigroups, with prehomomorphisms as morphisms, constitute a category. The category has products but not equalizers. 
In this paper we shall be primarily interested in prehomomorphisms of regular semigroups into inverse semigroups. The following theorem shows that, in a theoretical sense, the problem of constructing these reduces to that of constructing homomorphisms between inverse semigroups. It is the analog of McAlister (1976), Theorem 2.3 .

THEOREM 1.6. Let $S$ be a regular semigroup. Then there exist an inverse semigroup $I(S)$ and a prehomomorphism $\gamma=\gamma_{S}$ of $S$ into $I(S)$ with the following property:

if $\theta$ is any prehomomorphism of $S$ into an inverse semigroup $T$ then there is a unique homomorphism $\psi$ of $I(S)$ into $T$ such that the diagram

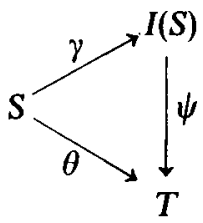

commutes.

ProOF. First note that $\theta: S \rightarrow T$ is a prehomomorphism if and only if

$$
\text { (ab) } \theta=(a b) \theta((a b) \theta)^{-1} a \theta b \theta
$$

for all $a, b \in S$.

Let $F=F I(S)$ be the free inverse semigroup on $S$ with $\eta$ the canonical embedding of $S$ into $F$ and let $\rho$ be the congruence on $F$ generated by the relations

$$
\text { (ab) } \eta=(a b) \eta(a b) \eta^{-1} a \eta b \eta \text {. }
$$

Set $I(S)=F / \rho$ and $\gamma=\eta \rho$. Then $\gamma$ is a prehomomorphism of $S$ into $I(S)$ and the universal property of $F$ shows that $\theta$ factors uniquely through $\gamma$.

Although Theorem 1.6 gives a theoretical method for constructing prehomomorphisms into inverse semigroups, it does not lead to a practical construction. For use later in the paper we shall need alternative methods for constructing prehomomorphisms of regular semigroups into inverse semigroups. The next lemma gives some elementary properties of such mappings.

LEMMA 1.7. Let $\varphi$ be a prehomomorphism of a regular semigroup $S$ into an inverse semigroup $T$. Then

(i) if $a$ and $b$ have a common inverse then $a \varphi=b \varphi$

(ii) if $x, y \in S a a^{\prime}$, where $a^{\prime} \in V(a)$, then $x \varphi=y \varphi$ if and only if $(x a) \varphi=(y a) \varphi$.

Proof. (i) is immediate since each element of an inverse semigroup has a unique inverse. 
(ii) Suppose $x \in S a a^{\prime}$ and let $x^{\prime} \in V(x)$. Then $x^{\prime} x \omega^{l} a a^{\prime}$ so that $(x a) \varphi=x \varphi a \varphi$. It follows that $x \varphi=y \varphi$ implies $(x a) \varphi=x \varphi a \varphi=y \varphi a \varphi=(y a) \varphi$.

Similarly, $(x a) \varphi=(y a) \varphi$ implies $\left(x a a^{\prime}\right) \varphi=\left(y a a^{\prime}\right) \varphi$ since

$$
x a, y a \in S a=S a^{\prime} a=S a^{\prime}\left(a^{\prime}\right)^{\prime}
$$

with $a=\left(a^{\prime}\right)^{\prime} \in V\left(a^{\prime}\right)$. But $x, y \in S a a^{\prime}$ so that we get $x \varphi=y \varphi$.

Theorem 1.6 shows that any prehomomorphism of a regular semigroup $S$ into an inverse semigroup $T$ can be factored through $\gamma_{S}$ and a homomorphism of $I(S)$ into $T$. However $I(S)$ is hard to construct, even if $S$ is inverse, so that in most cases, Theorem 1.6 does not give a practical method of constructing prehomomorphisms from regular to inverse semigroups. In some special cases, a situation dual to that in Theorem 1.6 prevails and the problem of constructing prehomomorphisms into inverse semigroups can be reduced to that of constructing prehomomorphisms between inverse semigroups. (A method of constructing some prehomomorphisms, in the general situation, is given in Construction 1.13.)

The following weak analog of the fundamental homomorphism theorem for semigroups will be crucial in obtaining the dual to Theorem 1.6.

LEMMA 1.8. Let $\varphi$ be a prehomomorphism of a regular semigroup $S$ into a regular: semigroup Tand let $\rho$ be a congruence on $S$ such that $\rho \subseteq \varphi \circ \varphi^{-1}$. Then $\varphi=\rho^{\natural} \theta$ for a unique prehomomorphism $\theta$ of $S / \rho$ into $T$.

Proof. For each $A \in S / \rho$, set $A \theta=a \varphi$ if $A=a \rho$. Then $\theta$ is the unique mapping of $S / \rho$ into $T$ such that $\varphi=\rho^{\natural} \theta$. Let $A=a \rho, B=b \rho$; then

$$
\text { (AB) } \theta=\left(a \rho^{\natural} b \rho^{\natural}\right) \theta=(a b) \rho \natural \theta=(a b) \varphi \leqslant a \varphi b \varphi=A \theta B \theta \text {. }
$$

Hence $\theta$ is a prehomomorphism.

THEOREM 1.9. Suppose that $S$ is either an orthodox or a completely regular semigroup and let $\mathscr{Y}$ be the minimum inverse semigroup congruence on $S$. Then each prehomomorphism $\varphi$ of $S$ into an inverse semigroup $T$ can be uniquely factored through $\mathscr{Y}$.

Proof. Suppose $S$ is orthodox. Then from Hall (1969)

$$
\mathscr{Y}=\{(a, b) \in S \times S: V(a) \cap V(b) \neq \varphi\} .
$$

Hence, if $\varphi$ is a prehomomorphism of $S$ into an inverse semigroup $T$, it follows, from Lemma 1.7 , that $\mathscr{Y} \subseteq \varphi \circ \varphi^{-1}$ and so, by Lemma $1.8, \varphi$ factors uniquely through $\mathscr{Y}$. Alternatively, suppose that $S$ is completely regular. Thus $S$ is the union of a

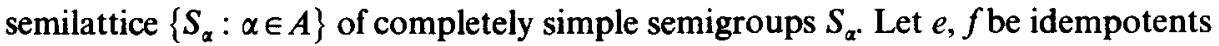
of $S_{\alpha}$. Then $e \mathscr{L} g \mathscr{R} f$ for some idempotent $g \in S_{\alpha^{*}}$. Thus, if $\varphi$ is a prehomomorphism of $S$ 
into an inverse semigroup $T$, then $\operatorname{e\varphi } \mathscr{L} g \varphi \mathscr{R} f \varphi$ so that, since $T$ is inverse, $e \varphi=f \varphi$. It follows that if $a \in H_{f}$ then $a \varphi \mathscr{H} f \varphi=e \varphi$ so that $\varphi$ maps the completely simple semigroup $S_{\alpha}$ into a single $\mathscr{H}$-class of $T$. Since it contains an idempotent, this $\mathscr{H}$ class $H_{\alpha}$ must be a group and then, since the natural partial order on a group is trivial, $\varphi$ must actually induce a homomorphism of $S_{\alpha}$ into $H_{\alpha}$.

For each $\alpha \in A$, let $U_{\alpha}=S_{\alpha} \varphi$ and let $U$ be the disjoint union of the $U_{\alpha}, \alpha \in A$. We shall show that $U$ can be turned into an inverse semigroup in such a way that $\varphi$ can be factored through a homomorphism of $S$ into $U$.

For each $\alpha \in A$, pick $e=e_{\alpha} \in S_{\alpha}$ and for each $\bar{b}=b \varphi \in U_{\beta}, \beta \geqslant \alpha$ set $\bar{b} \eta_{\beta, \alpha}=b \varphi e \varphi$. Then $e \varphi=f \varphi$ where $f \in S_{\alpha}$ is an idempotent with $f \omega b^{-1} b$. Hence $b \varphi е \varphi=b \varphi f \varphi=(b f) \varphi$ since $f \omega b^{-1} b=b b^{-1}$. But $b \varphi e \varphi \geqslant(b e) \varphi$ and, since be $\mathscr{D} e$, (be) $\varphi \mathscr{D} e \varphi \mathscr{D}(b f) \varphi$. Because the natural partial order on a group is trivial it follows from the inequalities

$$
b \varphi e \varphi=(b f) \varphi \geqslant(b e) \varphi,
$$

with all three terms in the same $\mathscr{H}$-class, that $b \varphi e \varphi=(b f) \varphi=(b e) \varphi$. Indeed, we have

$$
b \varphi e \varphi=(b e) \varphi=(e b) \varphi=e \varphi b \varphi \in U_{x}
$$

so that $\eta_{\beta, \alpha}$ is a mapping of $U_{\beta}$ into $U_{\alpha}$; it is, clearly, well defined.

Let $\bar{a}=a \varphi, \bar{b}=b \varphi \in U_{\beta}$. Then $\overline{a b}=(a b) \varphi$ since $\varphi$ is a homomorphism on $S_{\beta}$, so

$$
\begin{aligned}
(\overline{a b}) \eta_{\beta, \alpha}=(a b) \varphi e \varphi & =(a b) \varphi g \varphi \quad\left(\text { where } g \in S_{\alpha}, g \omega b^{-1} b=b b^{-1}\right) \\
& =(a b g) \varphi \quad\left(\text { since } a b \mathscr{L} b \text { and } g \omega b^{-1} b\right) \\
& \leqslant a \varphi(b g) \varphi \\
& =a \varphi[e \varphi(b g) \varphi] \quad\left(\text { since }(b g) \varphi \in U_{\alpha}\right) \\
& =\bar{a} \eta_{\beta, \alpha} \bar{b} \eta_{\beta, \alpha}
\end{aligned}
$$

so $\eta_{\beta, \alpha}$ is a prehomomorphism of the group $U_{\beta}$ into the group $U_{\alpha}$ and is therefore a homomorphism. Further, it is easy to see that the $\eta_{\beta, \alpha}$ form a directed set of homomorphisms and it therefore follows that $U$ becomes an inverse semigroup (semilattice of groups) under the product

$$
\bar{a} * \bar{b}=\bar{a} \eta_{\alpha, \alpha \wedge \beta} \bar{b} \eta_{\beta, \beta \wedge \alpha} \text { for } \bar{a} \in U_{\alpha}, \bar{b} \in U_{\beta} .
$$

Define $\varphi^{*}: S \rightarrow U$ by

$$
a \varphi^{*}=a \varphi \text { in } U_{\alpha} \text { if } a \in S_{\alpha}
$$


Then, for $a \in S_{a}, b \in S_{\beta}$

$$
\begin{aligned}
(a b) \varphi^{*} & =(a h . h b) \varphi^{*} & & \left(\text { where } h \in S\left(a^{-1} a, b b^{-1}\right)\right) \\
& =(a h . h b) \varphi & & \text { (in } \left.S_{\alpha \wedge \beta} \text { since } a h, h b \in S_{\alpha \wedge \beta}\right) \\
& =(a h) \varphi .(h b) \varphi & & \text { (since } \varphi \text { is a homomorphism on } S_{\alpha \wedge \beta} \text { ) } \\
& =a \varphi h \varphi . h \varphi b \varphi & & \text { (since } \left.h \omega^{r} a^{-1} a, h \omega^{\prime} b b^{-1}\right) \\
& =a \varphi^{*} \eta_{\alpha, \alpha \wedge \beta} b \varphi^{*} \eta_{\beta, \alpha \wedge \beta} & & \text { (since } \left.h \in S_{\alpha \wedge \beta} \text { and } h \varphi b \varphi=b \varphi h \varphi\right) \\
& =a \varphi^{*} * b \varphi^{*} . & &
\end{aligned}
$$

Thus $\varphi^{*}$ is a homomorphism of $S$ into the inverse semigroup $U$ and, from the definition $\varphi^{*} \circ \varphi^{*-1} \subseteq \varphi \circ \varphi^{-1}$. Hence $\mathscr{Y} \subseteq \varphi \circ \varphi^{-1}$ and so, by Lemma $1.9, \varphi$ can be uniquely factored through $\mathscr{Y}$.

Remark 1.10. The dual of Theorem 1.6 (analog of Theorem 1.9) is not true in general. Example 5.4 shows that a completely 0-simple semigroup may admit nontrivial prehomomorphisms into inverse semigroups even though it admits no nontrivial inverse homomorphic images.

REMARK 1.11. Because the category of regular semigroups and prehomomorphisms admits products a weak analog of Theorem 1.6 holds for prehomomorphisms in the following sense. Given any regular semigroup $S$ there exists an inverse semigroup $W$ and a prehomomorphism $\delta$ of $S$ into $W$ with the property that if $\theta$ is a prehomomorphism of $S$ into any inverse semigroup $T$ then there is a prehomomorphism $\psi: W \rightarrow T$ such that the diagram

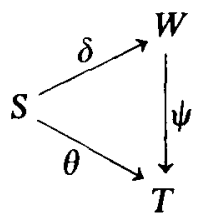

commutes.

Theorem 1.9 shows that, in case $S$ is orthodox or completely regular, then $W$ can be chosen as $\boldsymbol{S} / \mathscr{Y}$ and then $\psi$ is unique. I have been unable to show that $W$ can always be chosen so that $\psi$ is unique.

Theorem 1.9 gives a method for constructing prehomomorphisms when $S$ is orthodox or completely regular. For use later in the paper we need a method for constructing prehomomorphisms when $S$ is an arbitrary regular semigroup.

It follows from Lemma 1.7 that the equivalence relation $\rho=\varphi \circ \varphi^{-1}$ determined a prehomomorphism $\varphi$ of a regular semigroup $S$ into an inverse semigroup $T$ has the 
following properties :

(i) $V(a) \cap V(b) \neq \varphi$ implies $(a, b) \in \rho$;

(ii) $\left(x a a^{\prime}, y a a^{\prime}\right) \in \rho$ if and only if $(x a, y a) \in \rho$ for all $a, b, x, y \in S, a^{\prime} \in V(a)$.

Conversely, we shall show, given an equivalence relation $\rho$ on $S$, which satisfies (i) and (ii), how to construct a prehomomorphism from $S$ into an inverse semigroup. In order to simplify terminology in this construction, and later, we make the following definition.

DEFINITION 1.12. Let $S$ be a regular semigroup. Then an equivalence $\rho$ on $S$ is a right precongruence if

(i) $V(a) \cap V(b) \neq \emptyset$ implies $(a, b) \in \rho$;

(ii) $\left(x a a^{\prime}, y a a^{\prime}\right) \in \rho$ if and only if $(x a, y a) \in \rho$

for all $a, b, x, y \in S, a^{\prime} \in V(a)$.

CONSTRUCTION 1.13. Let $\rho$ be a right precongruence on a regular semigroup $S$ and, for $a \in S$, denote the $\rho$-class of $a$ by $[a]$.

For each $a \in S$ define $\rho_{a} b y$

$$
\Delta \rho_{a}=\left\{[x]: x \in S a a^{\prime}, a^{\prime} \in V(a)\right\}
$$

and

$$
[x] \rho_{a}=[x a] \text { for }[x] \in \Delta \rho_{a} \text {. }
$$

Then $\varphi=\varphi_{\rho}: a \rightarrow \rho_{a}$ is a prehomomorphism of $S$ into the symmetric inverse semigroup $\mathscr{I}_{S / \rho}$ on $S / \rho$.

Proof. First, we show that each $\rho_{a}$ is well defined. Let $a^{\prime}, a^{\prime \prime} \in V(a)$ and $x \in S$. Choose $x^{\prime} \in V(x)$ and $g \in S\left(x^{\prime} x, a a^{\prime}\right)$; then $a a^{\prime} g x^{\prime} \in V\left(x a a^{\prime}\right)$. But

$$
x a a^{\prime \prime} a a^{\prime} g x^{\prime} \cdot x a a^{\prime \prime}=x a a^{\prime} g x^{\prime} x a a^{\prime} \cdot a a^{\prime \prime}=x a a^{\prime} \cdot a a^{\prime \prime}=x a a^{\prime \prime}
$$

since $a a^{\prime} g x^{\prime} \in V\left(x a a^{\prime}\right)$. Similarly

$$
a a^{\prime} g x^{\prime} . x a a^{\prime \prime} . a a^{\prime} g x^{\prime}=a a^{\prime} g x^{\prime}
$$

so that $V\left(x a a^{\prime}\right) \cap V\left(x a a^{\prime \prime}\right) \neq \emptyset$. It follows that $x a a^{\prime} \rho x a a^{\prime \prime}$ so that

$$
\left\{[x]: x \in S a a^{\prime}\right\}=\left\{[x]: x \in S a a^{\prime \prime}\right\} .
$$

Hence the domain of $\rho_{a}$ is independent of the choice of $a^{\prime} \in V(a)$.

Suppose now that $[x]=[y]$ where $x, y \in S a a^{\prime}$. Then, by (ii), $[x a]=[y a]$ so that $\rho_{a}$ is well defined. On the other hand, if $[x] \rho_{a}=[y] \rho_{a}$ for $x, y \in S a a^{\prime}$ then $[x a]=[y a]$ implies $[x]=[y]$ by (ii). Hence $\rho_{a}$ is one-to-one and so is in $\mathscr{I}_{S / \rho}$. 
Let $a, b \in S, a^{\prime} \in V(a), b^{\prime} \in V(b), h \in S\left(a^{\prime} a, b b^{\prime}\right)$. Then $b^{\prime} h a^{\prime} \in V(a b)$ and so

$$
\Delta \rho_{a b}=\left\{[x]: x \in S a b b^{\prime} h a^{\prime}=S a h a^{\prime}\right\} .
$$

Let $x \in$ Saha'. Then $[x] \in \Delta \rho_{a}$ and $[x] \rho_{a}=[x a]$. But $x a=\left(x a h a^{\prime}\right) a=x a h$ since $x \in S a h a^{\prime}$ and since $h \in S\left(a^{\prime} a, b b^{\prime}\right)$. Let $x^{\prime} \in V(x), g \in S\left(x^{\prime} x\right.$, aha'); then $h a^{\prime} g x^{\prime} \in V(x a h)$. But

$$
\begin{aligned}
x a h b b^{\prime} . h a^{\prime} g x^{\prime} . x a h b b^{\prime} & =x a h . h a^{\prime} g x^{\prime} . x a h . b b^{\prime} \quad\left(\text { since } h \in S\left(a^{\prime} a, b b^{\prime}\right)\right) \\
& =x a h b b^{\prime} \quad\left(\text { since } h a^{\prime} g x^{\prime} \in V(x a h)\right) .
\end{aligned}
$$

Similarly

$$
\begin{aligned}
h a^{\prime} g x^{\prime} \cdot x a h b b^{\prime} . h a^{\prime} g x^{\prime} & =h a^{\prime} g x^{\prime} . x a h . h a^{\prime} g x^{\prime} \quad\left(\text { since } h \in S\left(a^{\prime} a, b b^{\prime}\right)\right) \\
& =h a^{\prime} g x^{\prime} \quad\left(\text { since } h a^{\prime} g x^{\prime} \in V(x a h)\right) .
\end{aligned}
$$

Thus $V(x a h) \cap V\left(x a h b b^{\prime}\right) \neq \emptyset$ so that, since $x a=x a h, h \in S\left(a^{\prime} a, b b^{\prime}\right)$,

$$
[x a]=[x a h]=\left[x a h b b^{\prime}\right]=\left[x a b b^{\prime}\right]
$$

so that $[x] \rho_{a} \in \Delta \rho_{b}$. Further

$$
[x] \rho_{a} \rho_{b}=[x a] \rho_{b}=\left[x a b b^{\prime}\right] \rho_{b}=[x a b]=[x] \rho_{a b} .
$$

It therefore follows that $\rho_{a b} \leqslant \rho_{a} \rho_{b}$ and so $\varphi$ is a prehomomorphism.

The set of right precongruences $\rho$ on a regular semigroup $S$ is clearly closed under intersections and therefore has a smallest member which we shall denote by $\pi$.

When $S$ is orthodox $\pi$ is the minimum inverse congruence on $S$. That is, $\pi$ is the smallest equivalence on $S$ which satisfies (i); it satisfies (ii) automatically. This is not the case in general. Example 5.1 shows that $\pi$ can properly contain the equivalence relation generated by

$$
\{(a, b) \in S \times S: V(a) \cap V(b) \neq \varphi\} .
$$

Construction 1.13 shows that every right precongruence $\rho$ on a regular semigroup $S$ gives rise to a prehomomorphism $\theta$ of $S$ into an inverse semigroup. In general, however, $\rho \neq \theta \circ \theta^{-1}$. Indeed

LEMMA 1.14. $a \theta=b \theta$ if and only if

(i) for all $x \in S a a^{\prime}\left[u \in S b b^{\prime}\right]$ there exist $y \in S b b^{\prime}\left[v \in S a a^{\prime}\right]$ such that

$$
(x, y) \in \rho[(u, v) \in \rho] \text {; }
$$

(ii) for all $x \in S a a^{\prime}, y \in S b b^{\prime},(x, y) \in \rho$ implies $(x a, y b) \in \rho$.

Proof. Suppose $a \theta=b \theta$ and let $x \in S a a^{\prime}$. Then $[x] \in \Delta a \theta=\Delta b \theta$ so that $(x, y) \in \rho$ for some $y \in S b b^{\prime}$. The dual also holds so (i) holds. Now suppose that $x \in S a a^{\prime}, y \in S b b^{\prime}$ 
and $(x, y) \in \rho$. Then $X=[x]=[y] \in \Delta a \theta=\Delta b \theta$ so that $[x a]=X a \theta=X b \theta=[y b]$. Thus (ii) holds.

Conversely, suppose that (i), (ii) hold for $a, b \in S$. Then from (i),

$$
\left\{[x]: x \in S a a^{\prime}\right\}=\left\{[y]: y \in S b b^{\prime}\right\}
$$

so that $\Delta a \theta=\Delta b \theta$. By (ii) $X a \theta=X b \theta$ for any $X \in \Delta a \theta=\Delta b \theta$.

We shall denote by $\rho^{*}$ the equivalence obtained from $\rho$ using (i) and (ii) in Lemma 1.14.

LemMA 1.15. Suppose that $\rho \subseteq \sigma$. Then $\rho^{*} \subseteq \sigma^{*}$.

ProOF. Let $(a, b) \in \rho^{*}$. Then clearly (i) of Lemma 1.13 implies (i) holds for $\sigma^{*}$. Next suppose $x \in S a a^{\prime}, y \in S b b^{\prime}$ and $(x, y) \in \sigma$. Since $(a, b) \in \rho^{*}$ there exists $u \in S b b^{\prime}$ such that $(x, u) \in \rho$; then $(u, y) \in \sigma$. Further $(x a, u b) \in \rho \subseteq \sigma$ and $(u b, y b) \in \sigma$ since $u, y \in S b b^{\prime}$. Thus $(x a, y b) \in \sigma$ so that $(a, b) \in \sigma^{*}$.

COROLLARY 1.16. Let $S$ be a regular semigroup and let $\pi$ be the smallest right precongruence on $S$. Then $\pi^{*}=\gamma \circ \gamma^{-1}$ where $\gamma$ is as in Theorem 1.6. It is the smallest equivalence on $S$ of the form $\theta \circ \theta^{-1}$ for a prehomomorphism $\theta$ of $S$ into an inverse semigroup.

\section{Fundamental regular semigroups and groups}

In this section we shall apply the machinery developed in Section 1 to investigate the extent to which a regular semigroup can be constructed from a fundamental semigroup and a group using direct products, regular subsemigroups and idempotent separating homomorphisms. To make matters precise we make the following definition.

DEFINITION 2.1. Let $S$ and $T$ be regular semigroups. Then $S$ divides $T$ strongly if there is an idempotent separating homomorphism from a full regular subsemigroup of $T$ onto $S$.

In the terminology of Definition 2.1, the main result of this section is the following.

THEOREM 2.2. Let $S$ be a regular semigroup. Then $S$ strongly divides the direct product of a fundamental regular semigroup and a group if and only if $\mu \cap \pi=\Delta$, where $\mu$ is the maximum idempotent separating congruence on $S$. 
Proof. We shall show that $S$ strongly divides the direct product of a group and a fundamental regular semigroup if and only if $\mu \cap \pi^{*}=\Delta$. This is equivalent to proving Theorem 2.2 , as shown by the following lemma.

LEMMA 2.3. Let $\rho$ be a right precongruence on a regular semigroup $S$. Then $\rho \cap \mathscr{H} \supseteq \rho^{*} \cap \mathscr{H}$. Thus $\pi \cap \mathscr{H}=\pi^{*} \cap \mathscr{H}$ and $\pi \cap \mu=\pi^{*} \cap \mu$.

Proof. Suppose that $(a, b) \in \rho^{*} \cap \mathscr{H}$. Then $a a^{\prime} \rho \in \Delta \rho_{a}=\Delta \rho_{b}$ so that

$$
a \rho=a a^{\prime} \rho_{a}=a a^{\prime} \rho_{b}=b \rho .
$$

Hence $(a, b) \in \rho \cap \mathscr{H}$.

Conversely, suppose $(a, b) \in \pi \cap \mathscr{H}$. Then clearly $(a, b) \in \pi^{*} \cap \mathscr{H}$. Hence $\pi \cap \mathscr{H}=\pi^{*} \cap \mathscr{H}$.

The equivalence $\pi^{*}$, and prehomomorphisms, appear in Theorem 2.2 because of the following considerations. Suppose that $T$ is a regular semigroup and $\theta, \psi$ are homomorphism of $T$ onto a regular semigroup $S$ and into a group $G$ respectively so that we have the following diagram of maps :

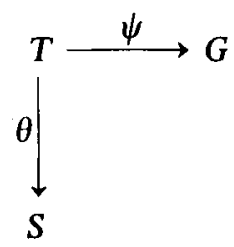

For each $s \in S$ set

$$
\begin{aligned}
s \varphi & =\{g \in G: t \theta=s, t \psi=g \text { for some } t \in T\} \\
& =s \theta^{-1} \psi .
\end{aligned}
$$

LEMMA 2.4. The mapping $\varphi: s \mapsto s \phi$ is a prehomomorphism of $S$ into the inverse semigroup $\mathscr{K}(G)$ of cosets of $G$.

Proof. Let $g, h, k \in s \varphi$ with, say $g=t \psi, h=u \psi, k=v \psi, t \theta=u \theta=v \theta=s$. Then, for

$$
u^{\prime} \in V(u), \quad u^{\prime} \psi=h^{-1} \quad \text { and } \quad u^{\prime} \theta \in V(u \theta)=V(s)
$$

Hence

$$
g h^{-1} k=\left(t u^{\prime} v\right) \psi \quad \text { and } \quad\left(t u^{\prime} v\right) \theta=s\left(u^{\prime} \theta\right) s=s
$$

so that $g h^{-1} k \in s \varphi$. Since, always, $s \varphi \subseteq s \varphi(s \varphi)^{-1} s \varphi$ it follows from this calculation that $s \varphi=s \varphi(s \varphi)^{-1} s \varphi$ and therefore that $s \varphi \in \mathscr{K}(\mathrm{G})$. 
Suppose now that $x, y \in S$ and let $g \in x \varphi, h \in y \varphi$ with $x=t \theta, y=u \theta$ where $t, u \in T$ and $t \psi=g, u \psi=h$. Then $g h=t u \psi, x y=t u \theta$ so that $g h \in x y \varphi$. Thus $x \varphi y \varphi \subseteq(x y) \varphi$. The natural partial order on $\mathscr{K}(G)$ is the inverse of inclusion so that $(x y) \varphi \leqslant x \varphi y \varphi$ in $\mathscr{K}(G)$. Hence $\varphi$ is a prehomomorphism of $S$ into $\mathscr{K}(G)$.

With these preliminaries we can move on to the proof of Theorem 2.1. Suppose that $S$ divides $F \times G$ strongly where $F$ is a fundamental regular semigroup and $G$ is a group. Then we have the following diagram of maps

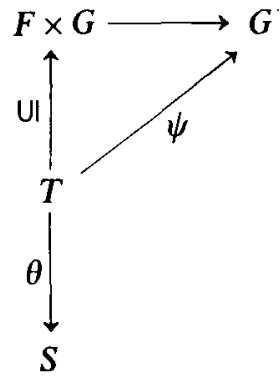

where $T$ is a full regular subsemigroup of $F \times G$ and the unnamed map is the projection onto $G$. By Lemma $2.3, \varphi=\theta^{-1} \psi$ is a prehomomorphism into $\mathscr{K}(G)$. Suppose that $(s, u) \in \mu \cap \pi^{*}$. Then, since $\mu \cap \pi^{*}=\mu \cap \pi \subseteq \varphi \circ \varphi^{-1}, s \varphi=u \varphi$ and so

$$
(a, g) \theta=s, \quad(b, g) \theta=u \text { for some } a, b \in F, \quad g \in G .
$$

Further since $\theta$ is idempotent separating $s \mu u$ implies $(a, g) \mu(b, g)$ in $T$. Thus, because $T$ is full in $F \times G,(a, g) \mu(b, g)$ in $F \times G$ and, finally, $a \mu b$ in $F$. Since $F$ is fundamental, this implies $a=b$ whence $s=u$. Therefore $\mu \cap \pi^{*}-=\Delta$.

On the other hand, suppose that $\mu \cap \pi^{*}=\Delta$ and let $\varphi$ be the prehomomorphism of $S$ into $\mathscr{I}_{S / \pi}$ arising from $\pi$ via Construction 1.13. Let $T^{\prime}=\langle S \varphi\rangle$, the (inverse) subsemigroup generated by $S \varphi$. By Lemma $0.4, T^{\prime}$ admits an $E$-unitary cover so that $T^{\prime}$ divides strongly $F \times G$ where $F$ is fundamental and $U$ is $E$-unitary with maximum group homomorphic image $G$, under the projection $F \times G \rightarrow G$ in the diagram

$$
\begin{aligned}
& F \times G \longrightarrow G \\
& \text { UI } \stackrel{\theta}{\longrightarrow} T^{\prime} \stackrel{\varphi}{ } \stackrel{ }{\longrightarrow} S .
\end{aligned}
$$

Consider

$$
T=\left\{(a, g) \in(S / \mu) \times G: a=s \mu, s \varphi=(b, g) \theta \text { for some } s \in S,(b, g) \theta \in T^{\prime}\right\} .
$$

Then, firstly, $T$ is a subsemigroup of $(S / \mu) \times G$. For, let $(a, g),(u, k) \in T$ where $a=s \mu$, $s \varphi=(b, g) \theta, u=t \mu, t \varphi=(c, k) \theta$. Then $a u=(s t) \mu$ and $s t \varphi \leqslant s \varphi t \varphi=(b c, g k) \theta$. But this implies $s t \varphi=e(b c, g k) \theta$ for some idempotent $e \in T^{\prime}$. Now $e=(f, 1) \theta$ for some idempotent $f \in F$ so that $s t \varphi=(f b c, g k) \theta$ so that $(a u, g k) \in T$. Next $T$ is regular. 
For if $(a, g) \in T \quad$ with $\quad a=s \mu, \quad s \varphi=(b, g) \theta \quad$ then $\quad\left(b^{-1}, g^{-1}\right) \in U \quad$ and $\left(b^{-1}, g^{-1}\right) \theta=((b, g) \theta)^{-1}=s^{\prime} \varphi$ for $s^{\prime} \in V(s)$ since $\varphi$ is a prehomomorphism. It follows that, if $a^{\prime}=s^{\prime} \mu$, then $\left(a^{\prime}, g^{-1}\right) \in T$ and is an inverse for $(a, g)$. Finally, if $e$ is an idempotent of $S / \mu$ then $e=f \mu$ for some $f^{2}=f \in S$. Now $f \varphi$ is idempotent in $T^{\prime}$ and so $f \varphi=(u, 1) \theta$ for some $u^{2}=u \in F$. It follows therefore that $(e, 1) \in T$ so that $T$ is a full regular subsemigroup of $(S / \mu) \times G$.

To complete the proof of the theorem, we need only construct an idempotent separating homomorphism of $T$ onto $S$. To this end, set

$$
(a, g) \psi=s \quad \text { if } a=s \mu, \quad s \varphi=(b, g) \theta \text { for some } b \in F .
$$

Suppose also that $a=t \mu, t \varphi=(c, g) \theta$ for some $c \in F$. Then $s \mu t$ implies $s \mathscr{H} t$ which in turn implies $s \varphi \mathscr{H} t \varphi$ and so, since $\theta$ is idempotent separating, $(b, g) \mathscr{H}(c, g)$. Since $(b, g)$ and $(c, g)$ have the same image, $g$, in the maximum group homomorphic image $G$ of $U$ which is $E$-unitary, it follows from these relations that $(b, g)=(c, g)$ so that $s \varphi=t \varphi$. Because $\mu \cap \pi^{*}=\Delta$, this means that $s=t$ so that $\psi$ is well defined; it is clearly onto.

Let $(a, g) \psi=s,(u, k) \psi=t$; thus $a=s \mu, s \varphi=(b, g) \theta, u=t \mu$ and $t \varphi=(c, k) \theta$ for some $b, c \in F$. Then the proof that $T$ is a subsemigroup of $(S / \mu) \times G$ shows that $s t \varphi=(f b c, g k) \theta$ for some idempotent $f \in F$, and, clearly, $s t \mu=a u$. Hence

$$
(a, g) \psi(u, k) \psi=s t=(a u, g k) \psi
$$

so that $\psi$ is a homomorphism. Finally, the idempotents of Tare the pairs $(e \mu, 1)$ with $e^{2}=e \in S$; since $(e \mu, 1) \psi=e, \psi$ is idempotent separating.

The method employed in the proof of Theorem 2.2 can also be used to determine when a regular semigroup strongly divides the direct product of a group and a combinatorial regular semigroup.

COROLlaRY 2.5. Let $S$ be a regular semigroup. Then $S$ strongly divides the direct product of a group and a combinatorial regular semigroup if and only if $\mathscr{H}$ is a congruence on $S$ and $\mathscr{H} \cap \pi=\Delta$.

Proof. Suppose that $S$ is an idempotent separating homomorphic image of $T^{\prime}$, where $T^{\prime}$ is a full regular subsemigroup of $T \times G$, with $G$ a group and $T$ combinatorial. Then $\mathscr{H}$ is a congruence on $T \times G$, thus on $T^{\prime}$. Hence, since $\theta$ is idempotent separating, $\mathscr{H}$ is a congruence on $S$. Thus, also, $\mathscr{H}=\mu$ and the conditions of Corollary 2.5 are necessary.

Conversely, suppose $\mathscr{H}$ is a congruence and $\mathscr{H} \cap \pi^{*}=\Delta$. Then $\mu=\mathscr{H}$ and so by the proof Theorem $2.2, S$ strongly divides the direct product of $S / \mu$ and a group. But, since $\mathscr{H}$ is a congruence, $\mu=\mathscr{H}$ so that $S / \mu=S / \mathscr{H}$ is combinatorial. 


\section{The equivalence $\pi$}

Theorem 2.2 reduces the problem of finding necessary and sufficient conditions, under which a semigroup $S$ strongly divides the direct product of a group and a fundamental inverse semigroup, to that of describing the equivalence relation $\pi$. In this section we give such a description which we can then use to strengthen Theorem 2.2 and Corollary 2.5. The first step in obtaining the description of $\pi$ is to introduce a class of equivalence relations on a regular semigroup $S$.

CONSTRUCTION 3.1. Let $S$ be a regular semigroup and let $U, V$ be full regular subsemigroups of $S$. Define a relation $\rho=\rho(U, V)$ on $S$ by $(a, b) \in \rho$ if and only if there exist idempotents $e, f \in S$, and elements $u \in U, v \in V$ such that a $\mathscr{R} \mathscr{L} u \mathscr{R} b, a \mathscr{L} f \mathscr{R} v \mathscr{L} b$ and $b=u a v$. Then $\rho$ is an equivalence on $S$ such that

$$
V(a) \cap V(b) \neq \varphi \quad \text { implies }(a, b) \in \rho .
$$

Iffurther, Vis self-conjugate in the sense that $a^{\prime} V a \subseteq V$ for each $a \in S, a^{\prime} \in V(a)$ then $\rho$ is a right precongruence on $S$.

Before verifying the statements made above it is helpful to give a diagrammatic representation of the $\mathscr{L}-\mathscr{R}$ relations holding between $a, e, f, u, v, b$. Here, as usual, vertical lines join $\mathscr{L}$-related elements while horizontal ones connect $\mathscr{R}$-related ones. Then $(a, b) \in \rho$ gives rise to the following diagram

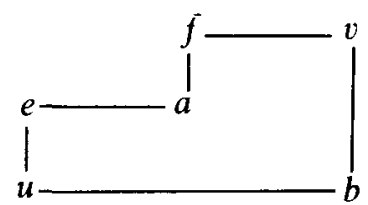

FigURE 1.

with $e, f$ idempotent and $u \in U, v \in V$.

VerifiCATION OF 3.1. Let $a \in S$. Then $a=\left(a a^{\prime}\right) a\left(a^{\prime} a\right)$ for $a^{\prime} \in V(a)$. Hence if we put $e=u=a a^{\prime}, f=v=a^{\prime} a$ we have the diagram in Figure 1 and also $a=u a v$. Thus $(a, a) \in \rho$ so $\rho$ is reflexive.

Next, let $(a, b) \in \rho$ and let $e, f, u, v$ be as in the description of the construction. Pick idempotents $g \in R_{b}, h \in L_{b}$. Then there exist $u^{\prime} \in V(u), v^{\prime} \in V(v)$ such that $e=u^{\prime} u$, $g=u u^{\prime}, f=v v^{\prime}, h=v^{\prime} v$, so that $b \mathscr{R} g \mathscr{L} u^{\prime} \mathscr{R} a, b \mathscr{L} h \mathscr{R} v^{\prime} \mathscr{L} a$ and $u^{\prime} \in U, v^{\prime} \in V$. Further, since $u^{\prime} u \mathscr{R} a, v v^{\prime} \mathscr{L} a$ we have

$$
a=u^{\prime} u a v v^{\prime}=u^{\prime} b v^{\prime}
$$

so that $(b, a) \in \rho$ and $\rho$ is symmetric. 
Next, suppose $(a, b) \in \rho,(b, c) \in \rho$. Then $b=u a v, c=w b z$ where $u, w \in U, \quad v, z \in V$ and $a \mathscr{R} e \mathscr{L} u \mathscr{R} b, a \mathscr{L} f \mathscr{R} v \mathscr{L} b, b \mathscr{R} g \mathscr{L} w \mathscr{R} c, b \mathscr{L} h \mathscr{R} z \mathscr{L}_{c}$

for idempotents $e, f, g, h \in S$. Now $c=w b z=(w u) a(v z)$ where $w u \in U, v z \in V$ and, since $u \mathscr{R} g \mathscr{L} w$ we have $u \mathscr{L} w u \mathscr{R} w$ and since $v \mathscr{L} h \mathscr{R} z$ we have $v \mathscr{R} v z \mathscr{L} z$. Thus

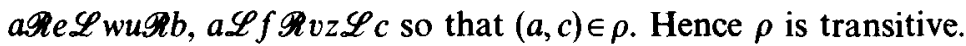

Suppose now that $a$ and $b$ in $S$ have a common inverse $x$. Then $b=b x b=(b x) a(x b)=u a v$ where $u=b x, v=x b$. Set $e=a x, f=x a$; then $e, f$ are idempotent as are $u, v$ (thus $u \in U, v \in V$, since $U, V$ are full) and $a \Re e \mathscr{L} \times \mathscr{L} u \mathscr{R} b$, $a \mathscr{L} f \mathscr{R} \times \mathscr{R} \cup \mathscr{L} b$. Thus $(a, b) \in \rho$. Finally, suppose that $V$ is selfconjugate and let $\left(x a a^{\prime}, y a a^{\prime}\right) \in \rho$ so that $y a a^{\prime}=u x a a^{\prime} v$ where $u \in U, v \in V$ and $x a a^{\prime} \mathscr{R} e \mathscr{L} u \mathscr{R} y a a^{\prime}$, $x a a^{\prime} \mathscr{L} f \mathscr{R} v \mathscr{L} y a a^{\prime}$ for some idempotents $e, f \in S$. Then $y a=(u x a)\left(a^{\prime} v a\right)$ where $u \in U$, $a^{\prime} v a \in V$.

Since $f \mathscr{L} x a a^{\prime}$ we have that $f=f a a^{\prime}$ which implies $a^{\prime} f a$ is idempotent. Further

$$
S x a=S f a=S f^{2} a=S f a a^{\prime} f a \subseteq S a^{\prime} f a \subseteq S f a
$$

so that $a^{\prime} f \mathscr{L} x a$. Likewise, $v \mathscr{L} y a a^{\prime}$ implies $v a \mathscr{L} y a$. But then

$$
S a^{\prime} v a \subseteq S v a=S v v^{\prime} v a=S f v a=S f a a^{\prime} v a \subseteq S a^{\prime} v a
$$

where $v^{\prime} \in V(v)$ is such that $v v^{\prime}=f=f a a^{\prime}$. Thus $a^{\prime} v a \mathscr{L} v a \mathscr{L} y a$. Hence $y a=u x a w$ where $u \in U, w \in V$ and $x a \mathscr{R} e \mathscr{L} u \mathscr{R} y a, x a \mathscr{L} a^{\prime} f a \mathscr{R} w \mathscr{L} y a$ so that $(x a, y a) \in \rho$.

It is easy to see that $\rho(U, V) \subseteq \rho\left(U^{\prime}, V^{\prime}\right)$ whenever $U \subseteq U^{\prime}, V \subseteq V^{\prime}$ so that there is a smallest equivalence of the form $\rho(U, V)$; namely $\rho(I G(S), I G(S))$ where $I G(S)$ denotes the subsemigroup generated by the idempotents of $S$. Likewise there is a largest equivalence of the form $\rho(\mathrm{U}, \mathrm{V})$; namely $\rho(S, S)$. The next lemma shows that $\rho(S, S)$ is, in fact, a very familiar object.

LEMMA 3.2. Let $S$ be a regular semigroup. The $\rho(S, S)$ is Green's relation $\mathscr{D}$. Further $\rho(S, S)^{*}$ is Green's relation $\mathscr{J}$.

Proof. First of all, if $(a, b) \in \rho=\rho(S, S)$ then $a \mathscr{R} e \mathscr{L} u \mathscr{R} b$ for some idempotent $e$ and element $u$ in $S$. Thus $a \mathscr{D} b$. Conversely, suppose that $a \mathscr{D} b$ and let $e, g$ be idempotents with $e \mathscr{R} a, g \mathscr{R} b$. Then there exists $u \in S, u^{\prime} \in V(u)$ such that $e=u^{\prime} u, g=u u^{\prime}$. Then

$$
b=g b=u u^{\prime} b=u u^{\prime} u u^{\prime} b=u a a^{\prime} u^{\prime} b=u a v,
$$

where $a^{\prime} \in V(a)$ is such that $e=a a^{\prime}$ and where $v=a^{\prime} u^{\prime} b$. Further, if $f=a^{\prime} a$ then $a \mathscr{L} \int \mathscr{R} v \mathscr{L} b$ so that $(a, b) \in \rho(S, S)$.

Now $(a, b) \in \rho^{*}=\rho(S, S)^{*}$ implies $\left(a a^{\prime}, y b b^{\prime}\right) \in \rho$ for some $y \in S, a^{\prime} \in V(a), b^{\prime} \in V(b)$. Thus $a \mathscr{D} a a^{\prime} \mathscr{D} y b b^{\prime}$. This implies $a \mathscr{F} y b b^{\prime}$ so that

$$
S a S \subseteq S y b b^{\prime} S=S y b S \subseteq S b S .
$$


Dually, $S b S \subseteq S a S$ so that $a \mathscr{J} b$. Conversely, suppose that $a \mathscr{J} b$ and let $x=x a a^{\prime} \in S a a^{\prime}$. Since $a \mathscr{J} b$ there exists $y \in S$ such that $y y^{\prime}=a a^{\prime}, y^{\prime} y \omega b b^{\prime}$. Then $x=x y y^{\prime}$ so that $x=x y y^{\prime} \mathscr{R} x y \in S b b^{\prime}$. Thus $(x, x y) \in \rho$ with $x y \in S b b^{\prime}$. Further, if $x \in S a a^{\prime}, y \in S b b^{\prime}$ and $x \mathscr{D} y$ then $x a \mathscr{R} x \mathscr{D} y \mathscr{R} y b$ so that $(x a, y b) \in \rho$. Hence $a \mathscr{J} b$ implies $(a, b) \in \rho^{*}$ so that $\rho^{*}=\mathscr{J}$.

Since $V(a) \cap V(b) \neq \emptyset$ implies $(a, b) \in \rho$ for each equivalence $\rho=\rho(U, V)$ it follows that $\pi_{0}$, the equivalence generated by

$$
\{(a, b) \in S \times S: V(a) \cap V(b) \neq \emptyset\}
$$

is contained in the smallest equivalence relation $\rho=\rho(U, V)$.

LemMA 3.3. Let $S$ be a regular semigroup. Then $\pi_{0}=\rho(I G(S), I G(S))$.

ProOF. By the remarks above, we need only show that $\rho=\rho(I G(S), I G(S)) \subseteq \pi_{0}$. Suppose that $(a, b) \in \rho$. Then there exist idempotents $e, f \in S$ and elements $u, v \in I G(S)$ such that $b=u a v$ and $a \mathscr{R} e \mathscr{L} u \mathscr{R} b, a \mathscr{L} f \mathscr{R} v \mathscr{L} b$. By a result of Nambooripad (1979) (Lemma 0.3) we may assume

$$
u=e_{2 n} e_{2 n-1}, \ldots, e_{1}, \quad v=f_{1} f_{2}, \ldots, f_{2 n}
$$

where $e_{i}, f_{i}$ are idempotent and

$$
e_{1} \mathscr{R} e_{2} \mathscr{L} e_{3}, \ldots, \mathscr{R} e_{2 n}, \quad f_{1} \mathscr{L} f_{2}, \ldots, \mathscr{L} f_{2 n} .
$$

Set $e_{0}=e, f_{0}=f, a=a_{0}$ and $a_{i+1}=e_{2 i+1} a_{i} f_{2 i+1}, 0 \leqslant i<n$. Then $a_{n}=b$. Further, for each $0 \leqslant i<n$ we have

$$
a_{i} \mathscr{R e} e_{2 i} \mathscr{L} e_{2 i+1} \mathscr{R} a_{i+1}, \quad a_{i} \mathscr{L} f_{2 i} \mathscr{R} f_{2 i+1} \mathscr{L} a_{i+1} .
$$

That is, $\left(a_{i}, a_{i+1}\right) \in \tau$ where

$$
\begin{aligned}
\tau=\{(x, y) \in S \times S: y= & u x v, x \mathscr{R} e \mathscr{L} u \mathscr{R} y \text { and } x \mathscr{L} f \mathscr{R} v \mathscr{L} y \text { for some } \\
& \text { idempotents } e, b, u, v \in S\} .
\end{aligned}
$$

Suppose now that $(x, y) \in \tau$ and let $e, f, u, v$ be as in the definition of $\tau$. Since $e \mathscr{R} x \mathscr{L} f$, $x$ has an inverse $x^{\prime}$ with $x x^{\prime}=e, x^{\prime} x=f$. But then $u \mathscr{L} e \mathscr{L} x^{\prime} \mathscr{R} f \mathscr{R} v$ so that

$$
\begin{aligned}
y x^{\prime} y & =u x v x^{\prime} u x v=u x x^{\prime} x v=u x v, \\
x^{\prime} y x^{\prime} & =x^{\prime} u x v x^{\prime}=x^{\prime} x x^{\prime}=x^{\prime} .
\end{aligned}
$$

Thus $x^{\prime} \in V(x) \cap V(y)$ so that $(x, y) \in \pi_{0}$. It follows that $\left(a_{i}, a_{i+1}\right) \in \pi_{0}, 0 \leqslant i<n$. Hence $(a, b)=\left(a_{0}, a_{n}\right) \in \pi_{0}$.

COROLlary 3.4. Let $S$ be a regular idempotent generated semigroup. Then $\pi=\pi_{0}=\mathscr{D}$. Further $\pi=\pi^{*}$ if and only if $\mathscr{J}=\mathscr{D}$ on $S$. 
Proof. By Lemma 3.3, $\pi_{0}=\rho(I G(S), I G(S))=\rho(S, S)=\mathscr{D}$, by Lemma 3.2. Since $S$ is self conjugate it follows from Construction 3.1 that $\pi \subseteq \mathscr{D}$. Hence since $\pi_{0} \subseteq \pi$ we have $\pi=\mathscr{D}$.

Finally, by Lemma $3.2, \mathscr{D}^{*}=\mathscr{J}$ so that $\pi=\pi^{*}$ if and only if $\mathscr{J}=\mathscr{D}$ on $S$.

There is a smallest self conjugate full regular subsemigroup of a regular semigroup $S$. We shall denote this by $C I G(S)$; it is the self conjugate closure of the subsemigroup $I G(S)$ of $S$ generated by the idempotents. Thus

$$
C I G(S)=\bigcup\left\{V_{n}: n \geqslant 0\right\},
$$

where $V_{0}=I G(S)$ and, for $i \geqslant 0$

$$
V_{i+1}=\left\langle a^{\prime} V_{i} a: a \in S^{1}, a^{\prime} \in V(a) \text { in } S^{1}\right\rangle .
$$

Note that, since $V_{0}$ is full, each $V_{i}$ is a full regular subsemigroup of $S$. By Construction $3.1, \rho=\rho(I G(S), C I G(S))$ has the property that

$$
\left(x a a^{\prime}, y a a^{\prime}\right) \in \rho \quad \text { implies }(x a, y a) \in \rho .
$$

Hence $\pi \subseteq \rho$. We shall show, conversely, that $\rho \subseteq \pi$ so that $\pi$ can be explicitly described as follows :

$(a, b) \in \pi$ if and only if there exist idempotents $e, f \in E$, elements $u, v \in C I G(S)$ such that $a \mathscr{R} \mathscr{L} u \mathscr{R} b, a \mathscr{L} f \mathscr{R} v \mathscr{L} b$ and $b=u a v$.

To show this, we shall need the following lemmas concerning $C I G(S)$.

LEMMA 3.5. Let $v=v_{1} \ldots v_{n}, n>1$, where $v_{j}=x_{j}^{\prime} u_{j} x_{j}, u_{j} \in V_{i}, x_{j}^{\prime} \in V\left(x_{j}\right), x_{j} \in S^{1}$ then $v=w z$ where

(i) $w \mathscr{L} e \mathscr{R} z$ for some idempotent $e \in S$,

(ii) $w \in x_{1}^{\prime} V_{i} x_{1}$, and

(iii) $z=z_{2} \ldots z_{n}$ where $z_{i} \in x_{i}^{\prime} V_{i} x_{i}, 2 \leqslant i \leqslant n$.

Proof. Suppose $n=2$ and let $h \in S\left(v_{1}^{\prime} v_{1}, v_{2} v_{2}^{\prime}\right)$ where $v_{j}^{\prime} \in V\left(v_{j}\right), j=1,2$. Then

$$
v=\left(v_{1} h\right)\left(h v_{2}\right)=w z \text { and } \quad w=v_{1} h \mathscr{L} h \mathscr{R} h v_{2}=z .
$$

Further $h \in S\left(v_{1}^{\prime} v_{1}, v_{2} v_{2}^{\prime}\right)$ implies $h=h v_{1}^{\prime} v_{1}$ so that, from the form of $v_{1}, h=h x_{1}^{\prime} x_{1}$. Thus

$$
w=v_{1} h=x_{1}^{\prime} u_{1} x_{1} h=x_{1}^{\prime} u_{1} x_{1} h x_{1}^{\prime} x_{1}=x_{1}^{\prime}\left(u_{1} x_{1} h x_{1}^{\prime}\right) x_{1} \in x_{1}^{\prime} U_{i} x_{1}
$$

since $h=h x_{1}^{\prime} x_{1}$ implies $x_{1} h x_{1}^{\prime}$ is idempotent and $U_{i}$ is full. Likewise $z \in x_{2}^{\prime} U_{i} x_{2}$ so that the result is true for $n=2$. 
Suppose that it is true for $n$ and let $v=v_{1} v_{2}, \ldots, v_{n+1}$ where $v_{j} \in x_{j}^{\prime} V_{i} x_{j}$. Then, by induction, $v_{2} \ldots v_{n+1}=t y$ where $t \mathscr{L} k \mathscr{R} y$ for some $k^{2}=k, t \in x_{2}^{\prime} V_{i} x_{2}$ and $y=y_{3} \ldots y_{n+1}$ where $y_{j} \in x_{j}^{\prime} V_{i} x_{j}, 3 \leqslant j \leqslant n+1$. Thus $v_{2} \ldots v_{n+1} \mathscr{R} t$.

Let

$$
h \in S\left(v_{1}^{\prime} v_{1}, t t^{\prime}\right)=S\left(v_{1}^{\prime} v_{1},\left(v_{2} \ldots v_{n+1}\right)\left(v_{2} \ldots v_{n+1}\right)^{\prime}\right)
$$

where $v_{1}^{\prime} \in V\left(v_{1}\right), t^{\prime} \in V(t)$. Then $v=\left(v_{1} h\right)(h t y)$. Then, as in the first paragraph, $w=v_{1} h \in x_{1}^{\prime} V_{i} x_{1}$ and likewise $h t \in x_{2}^{\prime} V_{i} x_{2}$. Thus $z=h t y=y_{2} \ldots y_{n+1}$ where

$$
y_{j} \in x_{j}^{\prime} V_{i} x_{j}, \quad 2 \leqslant j \leqslant n+1 .
$$

Further, since $t y \mathscr{R} t, z=h t y \mathscr{R} h t \mathscr{R} h \mathscr{L} v_{1} h=w$ as $h \in S\left(v_{1}^{\prime} v_{1}, t t^{\prime}\right)$. Hence the lemma is true for $n+1$ and therefore holds for all $n>1$.

The lemma can immediately be redefined to show that each element of $V_{i+1}$ can be written in the form $v_{1} v_{2} \ldots v_{n}$ where $v_{j} \in x_{j}^{\prime} V_{i} x_{j}$ and $v_{j} \mathscr{L} e_{j} \mathscr{R} v_{i+1}$ for some idempotent $e_{i}, 1 \leqslant i<n$. However, we shall not need this result.

For each $i \geqslant 1$, let $\rho_{i}=\rho\left(I G(S), V_{i}\right)$. Then it is immediate that

$$
\pi_{0}=\rho_{0} \subseteq \rho_{1} \subseteq \ldots \text { and } \rho\left(I G(S), C I G(S)=\cup\left\{\rho_{i}: i \geqslant 1\right\} .\right.
$$

We shall show by induction that each $\rho_{i} \subseteq \pi$, so that $\rho \subseteq \pi$. Then since the converse inclusion is clear we shall have shown $\rho=\pi$.

Suppose that $(a, b) \in \rho_{i+1}$. Then there exist idempotents $e, f \in S$ and elements $u \in I G(S), v \in V_{i+1}$ such that $a \mathscr{R} e \mathscr{L} u \mathscr{R} b, a \mathscr{L} f \mathscr{R} v \mathscr{L} b$ and $b=u a v$.

LeMma 3.6. Suppose that $\rho_{i} \subseteq \pi$ and that, with the notation above, $v=x^{\prime} w x$ where $w=x x^{\prime} w=w x x^{\prime} \in V_{i}$. Then $(a, b) \in \pi$.

Proof. We show first that $\left(a x^{\prime}, b x^{\prime}\right) \in \rho_{i}$. Indeed, since $b=u a v$ we have

$$
b x^{\prime}=u a v x^{\prime}=u a x^{\prime} w x x^{\prime}=u a x^{\prime} w
$$

where $u \in I G(S), w \in V_{i}$ so that it suffices to find idempotents $g, h$ such that $a x^{\prime} \mathscr{R} g \mathscr{L} u \mathscr{R} b x^{\prime}$ and $a x^{\prime} \mathscr{L} h \mathscr{R} w \mathscr{L} b x^{\prime}$.

Let $a^{\prime} \in V(a)$ be such that $a a^{\prime}=e, a^{\prime} a=f$. Then, since $v \mathscr{R} f$ we have $x^{\prime} x f=f$ and therefore $a x^{\prime} x a^{\prime} a x^{\prime}=a x^{\prime}$ so that $a x^{\prime} \mathscr{R} a x^{\prime} x a^{\prime}=e$ while $a x^{\prime} \mathscr{L} x f x^{\prime}$ and $x f x^{\prime}$ is idempotent. Put $g=e, h=x f x^{\prime}$. Then we need only show (i) $u \mathscr{R} b x^{\prime}$ and (ii) $h \mathscr{R} w \mathscr{L} b x^{\prime}$. Since $b=b x^{\prime} x$, because $b \mathscr{L} v=v x^{\prime} x$, (i) is in fact immediate so we turn to (ii).

Since $x^{\prime} x f=f$, we find $(x f) x^{\prime}(x f)=x f$ so that $x f \mathscr{R} x f x^{\prime}$. Thus

$$
w S=x v x^{\prime} S=x v x^{\prime} x S=x v S=x f S=x f x^{\prime} S
$$


since $v \mathscr{R} f$. Thus $w \mathscr{R} \times f x^{\prime}=h$. Finally, since $b \mathscr{L} v$,

$$
S b x^{\prime}=S v x^{\prime}=S x^{\prime} w x x^{\prime}=S x^{\prime} w=S x x^{\prime} w=S w
$$

since $w=x x^{\prime} w$. Thus $w \mathscr{L} b x^{\prime}$ and (ii) holds so that $\left(a x^{\prime}, b x^{\prime}\right) \in \rho_{i}$.

It follows that $\left(a x^{\prime}, b x^{\prime}\right) \in \pi$ and therefore $\left(a x^{\prime} x, b x^{\prime} x\right) \in \pi$. That is $\left(a x^{\prime} x, b\right) \in \pi$.

But, since $a^{\prime} a=f$ and $f=x^{\prime} x f$ we have

$$
\begin{aligned}
a x^{\prime} x \cdot a^{\prime} \cdot a \cdot x^{\prime} x & =a \cdot x^{\prime} x f x^{\prime} x=a f x^{\prime} x=a x^{\prime} x, \\
\left(a^{\prime} a x^{\prime} x\right) a^{\prime} & =\left(a^{\prime} a\right) x^{\prime} x\left(a^{\prime} a a^{\prime}\right)=a^{\prime} a x^{\prime} x f a^{\prime}=a^{\prime} a a^{\prime}=a^{\prime}
\end{aligned}
$$

so that $a^{\prime} \in V(a) \cap V\left(a x^{\prime} x\right)$. Hence $\left(a, a x^{\prime} x\right) \in \pi$. It follows therefore that $(a, b) \in \pi$.

THEOREM 3.7. Let $S$ be a regular semigroup. Then $\pi=\rho(I G(S), C I G(S))$.

Proof. As above, let $\rho_{i}=\rho\left(I G(S), V_{i}\right)$. Then

$$
\rho=\rho(I G(S), C I G(S))=\cup\left\{\rho_{i}, i \geqslant 0\right\}
$$

contains $\pi$. We use induction on $i$ to show that each $\rho_{i} \subseteq \pi$, whence $\rho \subseteq \pi$.

Since $\rho_{0}=\pi_{0} \subseteq \pi$ the result is certainly true for $i=1$. Suppose it is true for $i$ and suppose $(a, b) \in \rho_{i+1}$. Then there exist idempotents $e, f \in S$ and elements $u \in I G(S)$, $v \in V_{i+1}$ such that $a \mathscr{R e} \mathscr{L} u \mathscr{R} b, a \mathscr{L} f \mathscr{R} v \mathscr{L} b$ and $b=u a v$. Now $v=v_{1} v_{2} \ldots v_{n}$ where $v_{j} \in x_{j}^{\prime} V_{i} x_{j}, 1 \leqslant j \leqslant n$. By Lemma $3.6,(a, b) \in \pi$ if $n=1$. We use induction on $n$ to show this is true in general.

Suppose it is true for $n-1$. Then, without, loss of generality, we may assume, by Lemma 3.5, that $v_{1} \mathscr{L} h \mathscr{R} v_{2} \ldots v_{n}$ for some idempotent $h$. Let $a_{1}=e a v_{1}=a v_{1}$. Then $a \mathscr{R e} \mathscr{L} e \mathscr{R} a v_{1}=a_{1}$ and $a \mathscr{L} f \mathscr{R} v_{1} \mathscr{L} a v_{1}=a_{1}$ since $a \mathscr{L} f \mathscr{R} v \mathscr{R} v_{1}$. Hence $\left(a, a_{1}\right) \in \pi$ and it suffices to show that $\left(a_{1}, b\right) \in \pi$.

From the definition of $a_{1}$, we have $b=u a_{1} v_{2} \ldots v_{n}$. Further $a_{1} \mathscr{R e} \mathscr{L} u \mathscr{R} b$ so we need only find an idempotent $g$ such that $a_{1} \mathscr{L} g \mathscr{R} v_{2} \ldots v_{n} \mathscr{L} b$ in order to apply the induction hypothesis and conclude that $\left(a_{1}, b\right) \in \pi$. From Lemma 3.5, we have $a_{1} \mathscr{L} v_{1} \mathscr{L} h \mathscr{R} v_{2} \ldots v_{n}$. Further,

$$
S b=S v_{1} \ldots v_{n} \subseteq S v_{2} \ldots v_{n}=S v_{1} v_{2} \ldots v_{n}=S b
$$

since $v_{1} \mathscr{L} h \mathscr{R} v_{2} \ldots v_{n}$. Hence $a_{1} \mathscr{L} h \mathscr{R} v_{2} \ldots v_{n} \mathscr{L} b$ and we may take $g=h$. By the induction hypothesis, therefore, $\left(a_{1}, b\right) \in \pi$. Thus, since $\left(a, a_{1}\right) \in \pi$ we find $(a, b) \in \pi$.

It follows therefore, that $\rho_{i+1} \subseteq \pi$. Hence, by induction each $\rho_{i} \subseteq \pi$ so that $\rho=\pi$.

It is natural to ask under what conditions $\pi_{0}$ and $\pi$ coincide. The characterizations of these equivalences given in Lemma 3.3 and Theorem 3.7 permit this question to be answered. 
Proposition 3.8. Let $S$ be a regular semigroup. Then $\pi=\pi_{0}$ if and only if the subsemigroup generated by the idempotents is self conjugate.

Proof. If $I G(S)=C I G(S)$, then certainly $\pi=\pi_{0}$. Conversely, suppose that

$$
\rho(I G(S), U)=\rho(I G(S), V)
$$

we show that $U=V$. It follows from this that $\pi_{0}=\pi$ implies $I G(S)=C I G(S)$.

Let $b \in V$. Then, for $b^{\prime} \in V(b)$, set $e=u=b b^{\prime}=a=f, v=b$. Then $a \mathscr{R} e \mathscr{L} u \mathscr{R} b$, $a \mathscr{L} f \mathscr{R} v \mathscr{L} b$ and $b=u a v$ where $u \in I G(S), v \in V$. Thus $\left(b, b b^{\prime}\right) \in \rho(I G(S), V)$. On the other hand, if $a$ is idempotent and $(a, b) \in \rho(I G(S), V)$ then, since $V$ is full, $b \in V$. Hence,

$$
V=\{b \in S:(b, e) \in \rho(I G(S), V) \text { for some idempotent } e\} .
$$

Thus, $\rho(I G(S), U)=\rho(I G(S), V)$ implies $U=V$.

\section{Applications}

Now that we have a characterization of the equivalence $\pi$, we can give refinements of the main results of Section 2 which avoid the direct mention of equivalence relations.

THEOREM 4.1. Let $S$ be a regular semigroup. Then $S$ strongly divides the direct product of a group and a fundamental regular semigroup if and only if $C I G(S)$ is fundamental.

ProOF. Suppose that $C I G(S)$ is fundamental and suppose that $(a, b) \in \mu \cap \pi$. Then there exist elements $u \in I G(S), v \in C I G(S)$ and idempotents $e, f$ such that $a \mathscr{R} e \mathscr{L} u \mathscr{R} b$, $a \mathscr{L} f \mathscr{R} v \mathscr{L} b$ and $b=u a v$. Further, since $(a, b) \in \mu \subseteq \mathscr{H}, e \mathscr{H} u, f \mathscr{H} v$. Let $a^{\prime} \in V(a)$ be such that $a a^{\prime}=e, a^{\prime} a=f$. Then $\left(a a^{\prime}, u a v a^{\prime}\right) \in \mu$ where $a a^{\prime}, u a v a^{\prime} \in C I G(T)$ Thus, since $C I G(S)$ is fundamental $a a^{\prime}=u a v a^{\prime}$ so that $a=u a v a^{\prime} a=u a v=b$ since $v \mathscr{L} f=a^{\prime} a$. Hence $\mu \cap \pi=\Delta$ so that, by Theorem $2.2, S$ strongly divides the direct product of a group and a fundamental regular semigroup.

Conversely, suppose that $\theta$ is an idempotent separating homomomorphism of $T$ onto $S$ where $T$ is a full regular subsemigroup of $F \times G$ where $F$ is fundamental and $G$ is a group. From Lallement's Lemma (see Howie (1976), p. 52) it is easy to see that $C I G(T) \theta=C I G(S)$ and it is also easy to see that $C I G(T)$ is a full regular subsemigroup of $F \times\{1\}$ where $\{1\}$ denotes the identity of $G$. Thus $C I G(T)$ is fundamental and, since $\theta$ is idempotent separating, so is $C I G(S)$. 
COROLlARY 4.2. Let $S$ be an orthodox semigroup. Then $S$ strongly divides the direct product of a group and a fundamental orthodox semigroup.

In a similar fashion, one obtains the following result when fundamental is replaced by combinatorial.

THEOREM 4.3. Let $S$ be a regular semigroup. Then $S$ strongly divides the direct product of a group and a combinatorial regular semigroup if and only if $\mathscr{H}$ is a congruence on $S$ and $C I G(S)$ is combinatorial.

Corollary 4.4. Let $S$ be an orthodox semigroup. Then $S$ strongly divides the direct product of a group and a combinatorial orthodox semigroup if and only if $\mathscr{H}$ is a congruence on $S$.

COROLlary 4.5. Let $S$ be a union of groups. Then $S$ strongly divides the direct product of a group and a combinatorial regular semigroup if and only if $S$ is an orthodox band of groups.

When $S$ is finite, the result in Theorem 4.3 can be strengthened to give the following result.

THEOREM 4.6. Let $S$ be a finite regular semigroup. Then $S$ divides the direct product of a finite group and a finite combinatorial semigroup if and only if $\mathscr{H}$ is a congruence on $S$ and $C I G(S)$ is combinatorial.

Proof. By the constructions yielding Theorem 4.3, the conditions clearly suffice. Conversely, suppose that $S$ is a homomorphic image of a subsemigroup $T$ of the direct product of a finite group $G$ and a finite combinatorial semigroup $C$; let $\theta$ denote the homomorphism of Tonto $S$. By McAlister(1980), $\mathscr{H}$ is a congruence on $S$ so it suffices to show that $C I G(S)$ is combinatorial.

Let $U_{1}=I G(S)$ and $U_{n+1}=\left\langle a^{\prime} U_{n} a: a \in S^{1}\right\rangle$; then $C I G(S)=\bigcup\left\{U_{n}: n \geqslant 1\right\}$. Now, set $V_{1}=I G(T)$ and $V_{n+1}=\left\langle b^{\prime} V_{n} b: b \in T^{1}\right\rangle$, which is regular. We shall show firstly that $U_{n}=V_{n} \theta$ for each $n \geqslant 1$ and secondly that $V_{n} \subseteq C \times\{1\}$ for each $n$, so that $V_{n}$ is combinatorial. It follows that $V=\bigcup\left\{V_{n}: n \geqslant 1\right\}$ is combinatorial. Hence, since $V$ is finite, so is $C I G(S)=V \theta$.

Clearly, $V_{1} \theta \subseteq U_{1}$. On the other hand, the inverse image of an idempotent in $S$ contains an idempotent of $T$, since $T$ is finite. Hence $V_{1} \theta=U_{1}$. Suppose that $V_{n} \theta=U_{n}$; then, clearly, $V_{n+1} \theta \subseteq U_{n+1}$. On the other hand, if $a \in S, a^{\prime} \in V(a)$ then, by Rhodes (1966), Proposition 3.2, since $T$ is finite there exist inverse elements $b, b^{\prime} \in T$ such that $b \theta=a, b^{\prime} \theta=a^{\prime}$, so that

$$
a^{\prime} U_{n} a=b^{\prime} \theta V_{n} \theta b \theta=\left(b^{\prime} V_{n} b\right) \theta \text {. }
$$


Hence, since $U_{n+1}$ is generated by the sets $a^{\prime} U_{n} a, U_{n+1} \subseteq V_{n+1} \theta$. Thus $U_{n+1}=V_{n+1} \theta$ and $V \theta=C I G(S)$.

Again, since the idempotents of $T$ are of the form $(e, 1)$ when $e \in C$, it is immediate that $V_{1} \subseteq C \times\{1\}$. Suppose that $V_{n} \subseteq C \times\{1\}$ and let $v \in V_{n}, t \in T^{1}, t^{\prime} \in V(t)$. Then $v=(x, 1), \quad t=(y, g), \quad t^{\prime}=\left(y^{\prime}, g^{-1}\right) \quad$ where $\quad x, y, y^{\prime} \in C \quad$ and $\quad g \in G$. Thus $t^{\prime} v t=\left(y^{\prime} x y, 1\right) \in C \times\{1\}$ and, since $V_{n+1}$ is generated by $\bigcup\left\{t^{\prime} V_{n} t: t \in T^{1}, t^{\prime} \in V(t)\right\}$, it follows that $V_{n+1} \subseteq C \times\{1\}$. Hence $V \subseteq C \times\{1\}$.

Theorem 4.6 answers, for regular semigroups, the question of Schützenberger which was considered in McAlister (1980). The argument implicitly depends on the fact that every inverse semigroup admits an $E$-unitary cover. That is, if $S$ is an inverse semigroup then there is an $E$-unitary inverse semigroup $T$ and an idempotent separating homomorphism of $T$ onto $S$. Specifically, Theorem 2.2 uses the fact that $\mathscr{H} \cap \sigma=\Delta$ on $T$.

THEOREM 4.7. Let $S$ be a regular semigroup. Then there exist a regular semigroup $T$, on which $\sigma \cap \mathscr{H}=\Delta$, and an idempotent separating homomorphism $\theta$ of Tonto $S$, if and only if $C I G(S)$ is combinatorial.

ProOF. Suppose that $\sigma \cap \mathscr{H}=\Delta$ on $T$ and let $\theta$ be an idempotent separating homomorphism of $T$ onto $S$. Suppose that $w \in C I G(S)$. Then $w=u \theta$ where $u \in C I G(T)$. Since $u$ is a product of conjugates of products of conjugates of ... products of idempotents, $u \sigma=1$ in $T / \sigma$. Hence, since $\left(w^{2}, w\right) \in \mathscr{H}$ implies $\left(u^{2}, u\right) \in \mathscr{H}$, $w \in C I G(S)$ implies $\left(u^{2}, u\right) \in \mathscr{H} \cap \sigma$ and thus $u^{2}=u$ whence $w^{2}=w$. Thus $C I G(S)$ is combinatorial.

Conversely, suppose that $C I G(S)$ is combinatorial and let $\varphi$ be the prehomomorphism of $S$ into $\mathscr{I}_{S / \pi}$ associated with $\pi$. Let $T^{\prime}$ be an $E$-unitary cover of $\langle S \varphi\rangle$ with $\theta$ the idempotent separating homomorphism of $T^{\prime}$ onto $\langle S \varphi\rangle$. Then since $C I G(S)$ is combinatorial $\mathscr{H} \cap \pi^{*}=\mathscr{H} \cap \pi=\Delta$ so that, if we let

$$
T=\{(a, g) \in(S / \mu) \times G: a=s \mu, s \varphi=(c, g) \theta, \text { for some } s \in S\}
$$

then $T$ is a full regular subsemigroup of $(S / \mu) \times G$ and $\psi$, defined by $(a, g) \psi=s$ if $a=s \mu, s \varphi=(c, g) \theta$ for some $c \in S / \mu$, is an idempotent separating homomorphism of $T$ onto $S$ (see the proof of Theorem 2.2).

Suppose $((a, g),(b, h)) \in \sigma \cap \mathscr{H}$. Then, since $(a, g) \mapsto g$ is a homomorphism onto a group, $g=h$. Further $a \mathscr{H} b$. Thus, if

$$
a=s \mu, \quad b=t \mu, \quad s \varphi=(c, g) \theta \text { and } t \varphi=(d, h) \theta,
$$

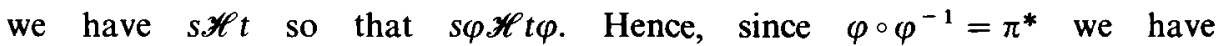
$(s, t) \in \mathscr{H} \cap \pi^{*}=\mathscr{H} \cap \pi=\Delta$. It follows that $a=b$ so that, since we have shown $g=h,(a, g)=(b, h)$. Hence $\sigma \cap \mathscr{H}=\Delta$ on $T$. 
As was the case with Theorem 4.3 , Theorem 4.7 can be strengthened when $S$ is finite.

THEOREM 4.8. Let $S$ be a finite regular semigroup. Then there exists a finite regular semigroup $T$, on which $\sigma \cap \mathscr{H}=\Delta$, and a homomorphism of $T$ onto $S$ if and only if $C I G(S)$ is combinatorial.

\section{Examples}

EXAMPLE 5.1. Let $S=\mathscr{M}(G, I, \Lambda ; P)$ be a compietely simple semigroup, and suppose, without loss of generality that $P$ has been normalized so that $p_{\lambda 1}=e=p_{1 i}$ for each $\lambda \in \Lambda, \quad i \in I$, where $e$ denotes the identity of $G$. Set $M=\{x \in G:(x, 1,1) \in I G(S)\}$. Then it is easy to see that $I G(S)=\{(x ; i, \lambda): x \in M, i \in I, \lambda \in \Lambda\}$ and so that since $I G(S)$ is full and completely simple, $M$ is a subgroup of $G$. Further, since the idempotents of $S$ have the form $\left(p_{\lambda i}^{-1}, i, \lambda\right), M$ is contained in the subgroup of $G$ generated by $\left\{p_{\lambda i}: i \in I, \lambda \in \Lambda\right\}$. On the other hand,

$$
\left(p_{\lambda i}^{-1}, 1,1\right)=(e, 1,1)\left(p_{\lambda i}^{-1}, i, \lambda\right)(e, 1,1) \in I G(S)
$$

so that $p_{\lambda i}^{-1} \in M$. Hence $M$ is the subgroup of $G$ generated by the entries of $P$.

Suppose that $M$ is not normal. Then there exists $x \in G$ such that $x^{-1} M x \nsubseteq M$. Hence,

$$
\left(x^{-1}, 1,1\right) I G(S)(x, 1,1)=\left(x^{-1} M x, 1,1\right) \nsubseteq I G(S)
$$

so that $C I G(S) \neq I G(S)$. It therefore follows that $\pi_{0} \neq \pi$.

EXAMPLE 5.2. Let $B$ be the fundamental $\omega$-simple semigroup with $d \mathscr{D}$-classes and let $w$ be the generator for the (cyclic) semigroup $L_{e}$ where $e$ denotes the identity of $B$. Let $P$ be the $2 \times 2$ matrix $\left[\begin{array}{ll}e & w \\ e & e\end{array}\right]$ and set $S=\mathscr{M}(B ; 2,2 ; P)$, the $2 \times 2$ matrix semigroup over $B$ with sandwich matrix $P$. Then $S$ is a combinatorial, idempotent generated simple regular semigroup with $d \mathscr{D}$-classes; each $\mathscr{D}$-class is isomorphic to the 4-spiral semigroup (Byleen and others (1978)).

Because $S$ is idempotent generated $\pi=\mathscr{D}$ and $\pi^{*}=\mathscr{J}$ so that $\pi \neq \pi^{*}$. Hence $\pi^{*} \neq \rho(U, V)$ for any full regular subsemigroups $U, V$.

EXAmple 5.3. Let $S=\mathscr{M}^{0}(G ; I, \Lambda ; P)$ be a completely 0-simple semigroup and let $N$ be the normal subgroup of $G$ generated by the non-zero entries of $P$; set $H=G / N$. 
Then the mapping $\varphi: S \rightarrow \mathscr{K}\left(H^{0}\right)$ defined by

$$
\begin{aligned}
(x ; i, \lambda) \varphi & =N x, \\
0 \varphi & =0,
\end{aligned}
$$

is easily seen to be a prehomomorphism of $S$ onto $H^{0}$.

Hence $S$ may admit nontrivial prehomomorphisms even though it is congruence free.

EXAMPLE 5.4. Let $S=\mathscr{M}^{0}(G ; I, \Lambda ; P)$ be a completely 0 -simple semigroup. Then, $S$ strongly divides the direct product of a fundamental regular semigroup and a group if and only if $C I G(S)$ is fundamental and thus combinatorial. In this case, $P$ can be normalized so that each entry of $P$ is either 0 or $e$, the identity of $G$.

On the other hand, suppose that each entry of $P$ is either $e$ or 0 . Then it is easy to show that each non-zero element of $C I G(S)$ has the form $(e, i, \lambda)$ so that $C I G(S)$ is combinatorial. Hence $S$ strongly divides the direct product of a group and a fundamental regular semigroup.

Define $i \sim j$ if and only if $p_{\lambda i} \neq 0, p_{\lambda, j} \neq 0$ for some $\lambda \in \Lambda$ and let $\equiv_{R}$ denote the transitive closure of $\sim$. Define $\equiv_{L}$ in a dual fashion. We can define a mapping $\theta: I \rightarrow \Lambda / \equiv_{L}$ as follows :

$$
i \theta=[\lambda] \quad \text { if } p_{\lambda i} \neq 0 \text { where }[\lambda] \text { denotes the } \equiv_{L} \text { class. }
$$

Then $\theta$ is well defined since $p_{\lambda i} \neq 0, p_{\mu i} \neq 0$ implies $\lambda \equiv_{L} \mu$. Further $i \sim j$ implies $i \theta=[\lambda]=j \theta$ for some $\lambda \in \Lambda$. Hence $\equiv_{R} \subseteq \theta \circ \theta^{-1}$ so that $\theta$ induces a mapping $\theta^{*}: I / \equiv_{R} \rightarrow \Lambda / \equiv_{L}$ defined by

$$
A \theta^{*}=B \quad \text { if } \exists i \in A, \lambda \in B \text { such that } p_{\lambda i} \neq 0 .
$$

Dually there is a mapping $\varphi^{*}: \Lambda / \equiv_{L} \rightarrow I / \equiv{ }_{R}$ defined by

$$
B \varphi^{*}=A \quad \text { if } \exists \lambda \in B, i \in I \text { such that } p_{\lambda i} \neq 0 .
$$

It is immediate that $\theta^{*}, \varphi^{*}$ are inverse mappings so that $\left|\Lambda / \equiv_{L}\right|=\left|I / \equiv_{R}\right|$.

Let $T$ be the Brandt semigroup $\mathscr{M}^{0}\left(G ; \Lambda / \equiv_{L}, \Lambda / \equiv_{L} ; \Delta\right)$ and define $\psi: S \rightarrow T$ by

$$
\begin{aligned}
(x, i, \lambda) \psi & =\left(x,[i] \theta^{*},[\lambda]\right), \\
0 \psi & =0 .
\end{aligned}
$$

Then, if $(x, i, \lambda)(y, j, \mu) \neq 0$ we have $p_{\lambda j} \neq 0$ so that

$$
\begin{aligned}
(x, i, \lambda) \psi(y, j, \mu) \psi & =\left(x,[i] \theta^{*},[\lambda]\right)\left(y,[j] \theta^{*},[\mu]\right) \\
& =\left(x y,[i] \theta^{*},[\mu]\right) \\
& =(x y, i, \mu) \psi=((x, i, \lambda)(y, j, \mu)) \psi
\end{aligned}
$$

since $p_{\lambda j} \neq 0$ implies $[\lambda]=[j] \theta^{*}$. Hence $\psi$ is a prehomomorphism of $S$ onto $T$. 
It can be shown that every prehomomorphism of $S$ into an inverse semigroup $W$ factors through a prehomomorphism of $T$ into $W$ via $\psi$.

The next two examples show that none of Theorems 4.1, 4.3, 4.7 remain true if $C I G(S)$ is replaced by $I G(S)$. The second example shows that this is also the case with Theorems 4.6, 4.8. Each of these examples involves a Rees matrix semigroup over an inverse semigroup and depends on the following simple properties of such semigroups. Let $T=T^{1}$ be an inverse semigroup, $I, \Lambda$ nonempty sets and $P$ a $\Lambda \times I$ matrix over $T$. Set $S=\mathscr{M}(T ; 1, \Lambda ; P)$.

1. $(x, i, \lambda)$ is idempotent if and only if $x \leqslant p_{\lambda i}^{-1}$. Suppose that $p_{\lambda 1}=1=p_{1 i}$ for each $i \in I, \lambda \in \Lambda$. Then $S$ is regular and

2. $I G(S)=\mathscr{M}(M ; 1, \Lambda ; P)$ where $M$ is the inverse subsemigroup of $T$ generated by

$$
\left\{x: x \leqslant p_{\lambda i}^{-1} \text { for some } i \in I, \lambda \in \Lambda\right\} .
$$

3. $(x, i, \lambda) \mathscr{H}(y, j, \mu)$ if and only if $i=j, \lambda=\mu$ and $x \mathscr{H} y$. Thus $\mathscr{H}$ is a congruence on $S$ if and only if it is a congruence on $T$.

EXAMPLE 5.5. Let $T=B(G, \theta)$ be a bisimple $\omega$-semigroup where $\theta$ is a non identity endomorphism of $G$. Thus $T$ has elements $(m, g, n), m, n \geqslant 0, g \in G$ with

$$
(m, g, n)(u, h, v)=\left(u \vee n-n+m, g \theta^{u \vee n-n} h \theta^{n \vee u-u}, n \vee u-u+v\right) .
$$

Let $w=(0,1,1)$ where 1 denotes the identity of $G$ and set $S=\mathscr{M}(T ; 2,2 ; P)$ where $P=\left[\begin{array}{ll}e & e \\ e & w\end{array}\right]$ with $e=(0,1,0)$ the identity of $T$.

Then, since $\mathscr{H}$ is a congruence on $T, \mathscr{H}$ is a congruence on $S$. Further $I G(S)=\mathscr{M}(M ; 2,2 ; P)$ where $M$ is the inverse subsemigroup of $T$ generated by $\left\{x: x \leqslant w^{-1}\right.$ or $\left.x \leqslant e\right\}$. Since $\left\langle w, w^{-1}\right\rangle$ contains all the idempotents of $T$, $M=\left\langle w, w^{-1}\right\rangle$ which is bicyclic and thus $I G(S)$ is combinatorial.

Let $z \in G$ be such that $z \neq z \theta$ and set $y=(0, z, 0) \in T$. Then $y^{-1}=\left(0, z^{-1}, 0\right)$. Let $x=(y, 1,1) \in S$; then $x^{\prime}=\left(y^{-1}, 1,1\right) \in V(x)$ so that, if we let $u=(w, 1,1) \in I G(S)$, we have $x^{\prime} u x \in C I G(S)$.

Now $x^{\prime} u x=\left(y^{-1} w y, 1,1\right)$ where $y^{-1} w y=\left(0, z^{-1}(z \theta), 1\right) \neq w$ so that $x^{\prime} u x \neq u$. Further, since $\mathscr{H}$ is a congruence on $T$ and $y \mathscr{H} 1, x^{\prime} u x \mathscr{H} u$. Hence, since $I G(S)$ is combinatorial, $x^{\prime} u x \notin I G(S)$. Consequently, $C I G(S) \neq I G(S)$ and $C I G(S)$ is not combinatorial. Since $\mathscr{H}$ is a congruence on $S$, it is a congruence on $\operatorname{CIG}(S)$ and hence, since $C I G(S)$ is not combinatorial, $C I G(S)$ is not a fundamental regular semigroup.

It follows that $I G(S)$ may be fundamental [combinatorial] without the same being true of $C I G(S)$, even if $\mathscr{H}$ is a congruence on $S$. 
EXAMPLE 5.6. Let $M_{2}$ denote the (inverse) semigroup of $2 \times 2$ matrix units and let $G$ be a finite non-abelian group. Let $T$ denote the quotient $\left(G \times M_{2}^{1}\right) /(G \times\{0\})$ of $G \times M_{2}^{1}$ by the ideal $G \times\{0\}$ and set $w=(y, 1,2)$ where $y$ is not in the centre of $G$. Consider $S=\mathscr{M}(T ; 2,2 ; P)$ where $P=\left[\begin{array}{ll}e & e \\ e & w\end{array}\right]$ with $e$ the identity of $T$. Then, since $\mathscr{H}$ is a congruence on $T$, it is a congruence on $S$ and $I G(S)=\mathscr{M}(M ; 2,2 ; P)$ where $M$ is the inverse subsemigroup of $T$ generated by $\{x \in T: x \leqslant e$ or $x \leqslant w\}$. From the structure of $T, M$ is thus $\left\{0, e, w, w^{-1}, w w^{-1}, w^{-1} w\right\} \approx M_{2}^{1}$ and so is combinatorial. Hence $I G(S)$ is combinatorial.

Now, let $z \in G$ be such that $y \neq z^{-1} y z(z$ exists since $y$ is not in the center of $G$ ) and set $x=(z, 1) \in T$. Then $x^{-1} w x=\left(z^{-1} y z, 1,2\right) \neq w$ and $x^{-1} w x \mathscr{H} w$. Set $u=(w, 1,1)$, $v=(x, 1,1)$; then $v^{\prime}=\left(x^{-1}, 1,1\right) \in V(v)$ so that $v^{\prime} u v \in C I G(S)$ but $v^{\prime} u v \neq u$ although, since $x^{-1} w x \mathscr{H} w, v^{\prime} u v \mathscr{H} u$. Hence, since $I G(S)$ is combinatorial, $v^{\prime} u v \notin I G(S)$. Consequently, $C I G(S)$ is not combinatorial. Since $\mathscr{H}$ is a congruence on $S$, it is a congruence on $C I G(S)$ and hence, since $C I G(S)$ is not combinatorial, it is not a fundamental regular semigroup.

It follows that $I G(S)$ may be fundamental [combinatorial] without the same being true of $C I G(S)$, even if $\mathscr{H}$ is a congruence on $S$ and $S$ is finite. Hence Theorems 4.6,4.8 are not true if $C I G(S)$ is replaced by $I G(S)$.

\section{References}

K. Byleen, J. Meakin and F. Pastijn (1978), 'The fundamental 4-spiral semigroup', J. Algebra 54, 6-26.

A. H. Clifford and G. B. Preston(1961), The algebraic theory of semigroups (Math. Surveys 7, Amer. Math. Soc., Providence, R.I.).

D. G. Fitz-Gerald (1972), 'On the inverses of products of idempotents in inverse semigroups', J. Austral. Math. Soc. 13, 335-337.

P. A. Grillet (1974), 'The structure of regular semigroups, I', Semigroup Forum 8, 177-187.

T. E. Hall (1969), 'On regular semigroups whose idempotents form a subsemigroup', Bull. Austral. Math. Soc. 1, 195-208.

T. E. Hall (1972), 'Congruences and Green's relations on regular semigroups', Glasgow Math. J. 13, 167-175.

T. E. Hall (1973), 'On regular semigroups', J. Algebra 24, 1-24.

J. M. Howie (1976), An introduction to semigroup theory (Academic Press, London).

D. B. McAlister (1974), 'Groups, semilattices and inverse semigroups', Trans. Amer. Math. Soc. 192, 227-244.

D. B. McAlister (1976), 'v-Prehomomorphisms on inverse semigroups', Pacific J. Math. 67, 215-231.

D. B. McAlister (1980), 'On a question of M. P. Schützenberger”, Proc. Edinburgh Math. Soc. (to appear).

D. B. McAlister and N. R. Reilly (1977), 'E-unitary covers for inverse semigroups', Pacific J. Math. 68, 161-174.

W. D. Munn (1970), 'Fundamental inverse semigroups', Q. J. Math. Oxford Ser. 21, 157-170.

K. S. S. Nambooripad (1979), 'The structure of regular semigroups, I', Mem. Amer. Math. Soc. 224. 
K. S. S. Nambooripad (1980), 'The natural partial order on a regular semigroup' (submitted). J. Rhodes (1966), 'Some results on finite semigroups', J. Algebra 4, 471-504.

B. M. Schein (1966), 'Semigroups of strong subsets' (Russian), Volž. Mat. Sb. 4, 180-186.

Department of Mathematical Sciences

Northern Illinois University

De Kalb, Illinois 60115

U.S.A. 